\title{
Adaptive Folk Theorization as a Path to Algorithmic Literacy on Changing Platforms
}

\author{
MICHAEL ANN DEVITO, University of Colorado Boulder, USA
}

\begin{abstract}
The increased importance of opaque, algorithmically-driven social platforms (e.g., Facebook, YouTube) to everyday users as a medium for self-presentation effectively requires users to speculate on how platforms work in order to decide how to behave to achieve their self-presentation goals. This speculation takes the form of folk theorization. Because platforms constantly change, users must constantly re-evaluate their folk theories. Based on an Asynchronous Remote Community study of LGBTQ+ social platform users with heightened self-presentation concerns, I present an updated model of the folk theorization process to account for platform change. Moreover, I find that both the complexity of the user's folk theorization and their overall relationship with the platform impact this theorization process, and present new concepts for examining and classifying these elements: theorization complexity level and perceived platform spirit. I conclude by proposing a folk theorization-based path towards an extensible algorithmic literacy that would support users in ongoing theorization.
\end{abstract}

CCS Concepts: •Human-centered computing Collaborative and social computing Empirical studies in collaborative and social computing •Human-centered computing $\sim$ Human computer interaction (HCI) Empirical studies in $\mathrm{HCI} \bullet$ Social and professional topics $\sim$ User characteristics

KEYWORDS: folk theories; folk theorization; algorithmic literacy; technology spirit; LGBTQ+

\section{ACM Reference format:}

Michael Ann DeVito. 2021. Adaptive Folk Theorization as a Path to Algorithmic Literacy on Changing Platforms. In Proceedings of the ACM on Human-Computer Interaction, Vol. 5, CSCW2, Article 339 (October 2021), 38 pages, https://doi.org/10.1145/3476080

\section{INTRODUCTION}

Imagine that you are a closeted queer person from an intolerant family, striking out on your own in a new city. The local queer Facebook community becomes your lifeline for everyday support. At the same time, while unsupportive, your family is still important to you, and mediating those relationships via Facebook provides the right mix of contact and distance to avoid conflict. For both cases, you must use Facebook, but you want to avoid context collapse and crossover between these audiences. You try to learn to manage how you're presenting yourself to each audience by reading some articles, and you form your own theory of how Facebook distributes your posts: Facebook

This work is supported by a Graduate Research Grant from The Graduate School at Northwestern University.

Author's addresses: Michael Ann DeVito, Department of Information Science, University of Colorado Boulder, $104518^{\text {th }}$ Street, UCB 315, Boulder, CO, USA.

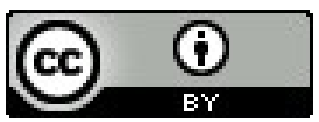

This work is licensed under a Creative Commons Attribution International 4.0 License.

(c) 2021 Copyright held by the owner/author(s).

2573-0142/2021/10-ART339. https://doi.org/10.1145/3476080

PACM on Human-Computer Interaction, Vol. 5, No. CSCW2, Article 339, Publication date: October 2021. 
processes the meaning of the post, and distributes it to people who seem interested in similar things based on their likes. You apply that theory regularly by making sure your queer posts have every tag and keyword needed to establish them as content Facebook would never think your family was interested in, and it works brilliantly - for a month, at which point Facebook changes something on their backend, and your strategy no longer works.

Our hypothetical user has an unusually high level of algorithmic awareness. She also presents herself in the heightened context of having a stigmatized identity, making these kinds of self-presentation decisions unusually salient to her [10,27, 43]. She was aware that the social platform she relied on for both benefit and protection was algorithmically-driven, and that accounting for this algorithm could be beneficial. Implicitly, she knew that to use the platform effectively - to achieve her self-presentation goals while avoiding pitfalls ranging from embarrassment [72] to stigmatization, harassment, and physical threats [27] - she had to decide how to use the visibility and audience management tools the platforms afforded her as part of the self-presentation process $[24,72]$. However, as social platforms are often opaque and hard to understand, making audiences difficult to predict or reliably target $[7,24,38]$, she had to rely on her folk theory for guidance in this decision-making.

Humans naturally try to form causal explanations of phenomena in the world [37, 61], broadly referred to as folk theories. In the context of HCI, folk theories are lay, sociallyconstructed conceptions of how a platform works, which the theorizer then uses to guide their on-platform decision-making behavior $[25,26]$. However, once a folk theory is formed and successfully deployed once, there is no guarantee it will work again. Social platforms, and especially the criteria by which algorithmically-driven content distribution mechanisms operate, are constantly in flux [38] and provide few cues for users as to when change has occurred and what the change is.

Despite the opaque environment and frequent change, users adapt (e.g., [26, 101]). For many, this is a necessity - social platforms, acting as the modern equivalent of a public square [41], have become deeply integrated into their personal and professional lives (e.g. [27, 30, 63]), even to the level of outright "delegation of human behaviour to algorithmic processes" [112 p. 139]. It is now a crucial life skill to be able to effectively use algorithmically-driven social platforms - as Cotter puts it, to "play the visibility game" [17]. To do that, users need folk theories that guide them in a useful direction.

While prior work provides some existing knowledge of this type of adaptation, it is restricted to the context of initial adaptation among professional or power users such as social media influencers [17] and digital entrepreneurs [63]. As work on more general populations [25, 26, $31,32,88$ ] focuses only on initial folk theory formation, we do not yet have a detailed understanding of the everyday process of repeated folk theorization and adaptation that casual, non-professional users employ to maintain their ability to effectively pursue their goals in the face of rapid and sometimes subtle changes to algorithmic systems. Deeper investigation in this area in the context of everyday users will further refine our understanding of both the personal folk theorization process and self-presentation process, making both more robust to change. Moreover, by deepening understanding in the specific context of self-presentation, a universal, constant [66] social process which is known to be impacted by algorithmic mechanisms [24], we can evaluate folk theorization's general utility as a tool that can be leveraged to start building and encouraging enhanced algorithmic understanding via an extensible literacy in this area. In turn, by focusing on an LGBTQ+ population who are more likely to notice and be negatively 
impacted by structural issues $[4,48]$ and for whom self-presentation has heightened importance $[10,27,43]$, we can capture both mainstream and outlier experiences.

In this paper, I present an Asynchronous Remote Community [73, 74, 75, 108] study of how non-expert, non-professional users notice, theorize, and adapt to change on algorithmicallydriven social platforms in the context of self-presentation. I perform a constructivist grounded theory [15] analysis of seven weeks of varied elicitation prompts and follow-up interviews with a group of 25 queer participants who have heightened, but not professional, self-presentation circumstances. I find that self-presentation-related adaptation in this context is impacted by the sophistication of the folk theorization the user is engaged in as well as the user's existing relationship to both the platform at large and the specific change in question. I contribute a new system for classifying folk theorization alongside a set of model adaptation pathways, a revised concept of technology spirit $[16,23]$ for the social platform era, updates to prior folk theorization and self-presentation models which account for platform change, and a new definition and direction for a future algorithmic literacy.

\section{BACKGROUND}

As algorithmically-driven platforms have become more and more embedded in our lives and power structures [7, 38, 40, 82, 112], calls for algorithmic accountability via increased algorithmic knowledge (e.g. [1, 83, 91]), and specifically "algorithmic literacy," have emerged $[20,91]$. Calls for literacy are often a response to new technologies which create power structures, and thus a need to understand and interact with those structures [71]. For algorithms and the systems they drive, this power shift has come from the structural ascendency of algorithmic authority into areas such as information curation, banking, housing, and even cultural production [7, 79, 91, 96, 104]. Considering the already-detectable gap in usable algorithmic knowledge along lines of education, age, and socioeconomic status [18], an increased focus on algorithmic literacy is essential as a bulwark against stripping users of their autonomy.

For guidance on how to approach algorithmic literacy, we can look to a precursor power shift, the proliferation of the internet itself. Researchers advocated for a focus on effective and efficient individual use instead of simple access [50]), setting the goal of bridging the digital divide by empowering individuals to not just consume, but instead intelligently participate in the new medium to accomplish their own goals [46]. Considering the threat to autonomy that algorithmically-driven platforms present [18], juxtaposed against the embeddedness and influence of these systems [7, 18, 25, 38, 40,112], I follow this example and take up a definition of algorithmic literacy based on Gurstein's definition of effective use for ICTs generally [46]: the capacity and opportunity to be aware of both the presence and impact of algorithmically-driven systems on self- or collaboratively-identified goals, and the capacity and opportunity to crystalize this understanding into a strategic use of these systems to accomplish said goals.

As an emerging technological literacy, it is also essential that algorithmic literacy focus on the context of the whole sociotechnical system in question, maintain a two-way relationship between literacy and technology, and approach understanding systems in a continuously critical way [71]. This last point is essential, as it reflects the ever-changing nature of algorithmically-driven platforms themselves [38]. Any algorithmic literacy must deal with this need to be constantly critical in the face of change. This requires moving beyond previous treatments of algorithmic literacy and related knowledge as static [63], as well as skills based "checklist" approaches (e.g., [51]) which, like past approaches to information literacy, focus on 
assessment of specific, limited skills at one point in time rather than an ongoing, extensible educational process [109]. It also requires being mindful that individuals are already immersed in the environment in question, making algorithmic literacy an exercise in mostly formalizing and correcting knowledge found in the world instead of purely introducing new knowledge [62]. These requirements point to a potential lens on algorithmic literacy which accounts for all of them: folk theorization.

\subsection{Folk Theories and Theorization}

Folk theories are intuitive, informal theories that reflect ideas about causal relationships [59]. In the specific context of algorithmically-driven social platforms, DeVito et al. define folk theories as "intuitive, informal theories that individuals develop to explain the outcomes, effects, or consequences of technological systems, which guide reactions to and behavior towards said systems" [26 p. 3165]. These theories explain how users deal with the complexity of platforms of which they have no direct technical knowledge [26].

Folk theories are an important part of how humans naturally learn about complex domains $[58,59]$. Human beings are naturally interested in mechanism but bad at understanding it [61], which results in us forming folk theories. Folk theories are also flexible enough to account for the uncertainty and instability inherent in human understandings of complex systems, in that they do not require full mechanistic detail or even dense mechanistic knowledge to be useful $[59,61]$, account for the fragmentary nature of non-expert understanding [37, 95], and can internally contradict each other in a relatively stable fashion [25, 32, 37, 59]. Folk theories let us meet the user where they are in terms of understanding and literacy, regardless of how contradictory, sparse, or fragmented these understandings may be, instead of attempting to start from scratch [62]. Additionally, folk theories are naturally malleable and changeable over time $[25,94,95]$, and may in fact improve with repeated rounds of theorization [94], making them compatible with the ever-changing nature of platforms.

In HCI, examining user folk theories is often a diagnostic technique, used to examine the extent to which users understand certain concepts, how they deal with certain problems, and how they accomplish discrete tasks. Both Eslami et al. and Rader et al. examined user folk theories of how content is delivered to them via algorithmically-driven social platform feeds, diagnosing an overall low level of algorithmic awareness in 2015-2016. Both research teams found distinct folk theories held by groups of users with associated consequences for platform use and design $[31,32,88]$. Bernstein et al. undertook more focused study in the same context to diagnose the extent to which and reasons why Facebook users misestimate their invisible audiences on the platform [9]. DeVito et al. also examined folk theories of curation by platforms, but in the specific context of a large-scale negative reaction to platform change to diagnose the nature and causes of the backlash [26]. French and Hancock, by contrast, focused less on mechanism and more on affect in their study of metaphorical folk theories of the influence of social platforms on content posting [34]. Regardless of their domain, these studies generally report out on specific, usually mechanistically-focused folk theories of specific technologies at one point in time - excellent for diagnosing problems in design, but of limited utility when attempting to move towards a sustainable, extensible literacy.

A second group of studies focus not on folk theories themselves, but the process of how users form these folk theories. For example, DeVito et al. examined how folk theories are formed in the context of self-presentation as instantiated through posting behavior, identifying not only how users form theories in this context, but how this process is embedded within and impacts 
the key social process of self-presentation [25]. It is this second type of folk theories approach a folk theorization approach - which I take in this project.

\subsection{Folk Theories and Self-Presentation}

Self-presentation (and more broadly impression management) is the constant and universal process by which we attempt to control how others view us $[42,66,103]$. Due to its ubiquity, the fact that it is highly impacted by algorithmic mechanisms [24], and the existence of a static version of its relevant folk theorization [25] process (Figure 1), it is an ideal process to focus on in order to examine adaptation to platform change.

According to Goffman's dramaturgical model of self-presentation, self-presentation is a twoway interplay between the self and one's audience, letting the self in question know what is and is not appropriate for this particular audience [42]. Social platforms, however, directly threaten this interplay; prior research has shown that the algorithmic mechanisms which control content distribution obscure one's audience, leaving few cues and little information on which to base self-presentation decisions [24]. For those that are in some way aware that there is an algorithmic mechanism at play, this gap in the self-presentation process is initially bridged by a folk theory of how the mechanism in question distributes content, often informed by endogenous (based on the platform itself, e.g. experimenting with the system) and/or exogenous (originating from outside the platform, e.g. from the news) information [25] (see Figure 1, top right).

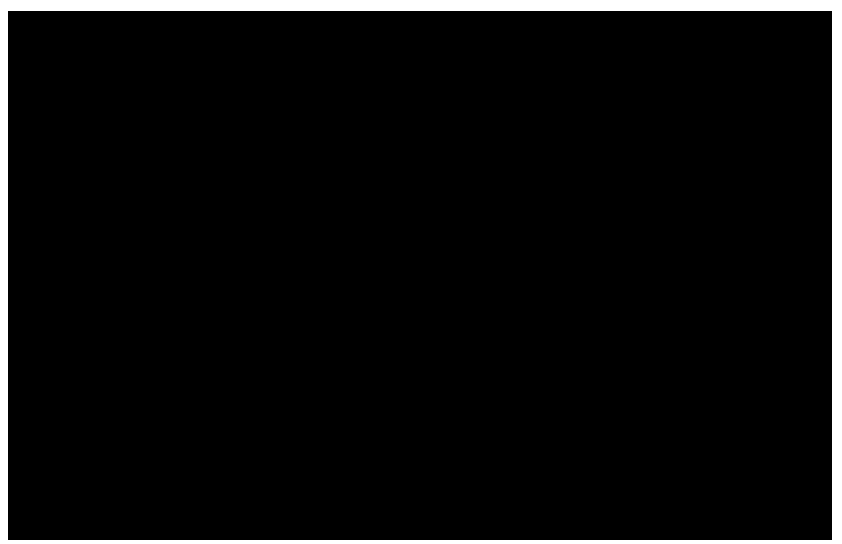

Figure 1: Folk theory formation process in the context of social media self-presentation on algorithmically-driven social platforms, reproduced from DeVito et al. [25].

However, the process of folk theorization is not activated unless there is at least some level of algorithmic awareness, and accounts of algorithmic awareness vary. Eslami et al. and Rader et al. posit that algorithmic awareness in the context of social platforms is quite low [32, 87, 88], while DeVito et al. advanced the idea that we do not adequately examine low-level (or "abstract") understandings of algorithms, and therefore underestimate awareness [26]. As such, I ask:

RQ1: To what extent are users aware of and theorizing about how algorithmicallydriven social platforms work? 


\subsection{Noticing Change}

Change challenges folk theories because the underlying mechanism the folk theory is attempting to explain shifts. This means the folk theory may no longer work as expected, and may in fact now mislead the user. Further complicating this is the fact that changes on social platforms are often unexpected, small in magnitude, not directly relevant to the user's own experience, and in the context of a system that is itself difficult to explain - all conditions which make change harder to notice [99]. Additionally, users may not understand "change" the same way a researcher or an engineer would. Recent work indicates that people are more aware of algorithms and the broad strokes of what they do than in the past and, despite the lack of mechanistic knowledge that academic studies have identified, have plenty of opinions about algorithmically-driven systems and the companies that control them [102], and often conflate algorithmic change with the actions of the platform company as a whole [26, 101].

In light of this, the heart of understanding change is focusing on perception - users adapt using the knowledge and perceptions they have. Drawing on recent work which suggests centering not just the user but their positionality and relational orientation when trying to understand user perceptions [5], I define "change" specifically from the vantage point of the user's perception, within the domain of self-presentation: any perceived difference relating to a social platform which the user believes may impact their use of or decision making around achieving their goals via said social platform. Importantly, I do not assert a difference between perceived changes that reflect real-world change and perceived changes that have no basis in real-world change. As seen in past studies, the truth of what is happening has minimal relevance to the user; they form folk theories, adapt, and even start protests with the knowledge they perceive $[25,26]$.

Ultimately, to adapt to change, one must first notice change, and understand that it is, in fact, a change. This crucial "noticing" step is the start of all sensemaking processes [110], including the already-established formation process for self-presentation-related folk theories of algorithmic systems [25] (Figure 1, top left). Taken together, I ask:

\section{RQ2: How and to what extent do users notice change on algorithmically-driven social platforms?}

\subsection{Deciding To Act (or Not)}

In most technology-related sensemaking processes, once change is noticed, users begin adapting by trying to apply their previous theory (here, a folk theory) of how a process or piece of technology works, "bracketing" (or bounding) what has actually changed (e.g., what seems to not work anymore about the present theory), applying previous knowledge to see if this change fits into a category of similar changes which would have similar solutions (labeling), and eventually creating a believable story about the change that can guide the subsequent reaction to the change [110]. However, this process is not guaranteed - not all users adapt in the face of change, or even continue using a technology.

One instructive model of adaptation we can draw on is Adaptive Structuration Theory (AST). Developed as a way to approach investigating the social and behavioral effects of technology in workplace settings, the core of AST is that existing structural features provide a bounding framework for how individuals will respond to changes in technology [23]. Here, "structural features" refers to the inputs to this process that can be said to exist "in the world" at the start of a sensemaking loop. This includes features such as technical structures (e.g. affordances) [23], individual psychological characteristics (e.g., personality factors), and prior knowledge (here, 
encapsulated as user's existing folk theory) [98], as well as the fit of the technology to the task a user is trying to perform [23]. It can also include the platform's spirit [76], which has been variously defined as the official claims made about a technology and what is communicated through design choices [23], a "property of the technology as it is presented to users" [76], and "a user's understanding of that technology's capabilities and affordances" [98]. While this list of structural features may not directly translate to the context of social platforms, it still represents a starting point for inquiry, allowing me to ask:

RQ3: How do users decide if and to what extent they will attempt to respond to change on algorithmically-driven social platforms?

\subsection{Adaptation \& Resistance}

Finally, once the decision to adapt has been made, action must actually be taken. Prior work suggests this process has two crucial components: how one comes to a decision on what actions to take, and then taking said action. In the folk theorization process established for selfpresentation, this would potentially include more information foraging (Figure 1, top half of loop) and sensemaking to refine one's folk theory in order decide on how to update one's actual behavioral tactics, and then a deployment of these tactics as updated self-presentation behaviors [25] (Figure 1, bottom half of loop).

Individual social and exploratory processes largely drive adaptation to technology in an organizational context $[6,23,98]$, and there is reason to believe this will be true in a personal social platform context as well. Consider prior work in a non-organizational, social platformbased, but still professional context. Instagram influencers have been shown to work collectively to identify and adjust for upcoming changes to the Instagram feed algorithm [17], while Airbnb hosts engage in strategies around updating the language and posting recency of their listings in an attempt to game the platform's search results based on forums and platform documentation [55]. Etsy store owners engage in a similar, but socially-mediated, process to keep their products showing up high in their platform's searches [63]. Considering this heightened parallel, and the foraging of both socially-sourced exogenous information and platform exploration-derived endogenous information in our existing model of folk theorization around self-presentation [25], it is reasonable to expect some kind of information-gathering process to proceed behavior change.

In terms of taking action, DeVito et al have argued that behavior flows from an updated folk theory [25]; the nature of the update to the behavior is not specified. In the context of change, instead of initial theory formation, it is necessary to expand our understanding to analyze behavioral impacts of platform change. In an organizational context, users adapt to systems by adjusting how they perform a task to fit the new technical reality, or by finding ways to use technology in new, often unintended ways to support the tasks as already performed [23, 98]. In turn, these adaptations can be faithful to the technology (based in the way the platform was designed to be used) or unfaithful (outside of the way the platform was designed to be used) [23]. The work on influencers and professionals noted above largely concerns faithful adaptations $[17,55,63]$, but we do not yet have a clear picture of how the nature of these adaptations in a personal, everyday use context. As such, I ask:

RQ4: How do users adapt to platform change on algorithmically-driven social platforms? 


\section{METHODS}

I adopted constructivist grounded theory [15] as my overall approach to allow the inductive generation of knowledge, based directly on a variety of participant experiences and perceptions. In turn, as constructivist grounded theory encourages the use of innovative, creative methods which are responsive to theoretical developments in the field [15], I adopted Asynchronous Remote Communities as my framework method.

Asynchronous Remote Communities (ARC) are an online, distributed framework for research that uses a secret social media group to deploy weekly prompts that engage participants on our key questions using multiple elicitation modalities [73, 75, 86]. ARC was initially developed in the context of health informatics $[73,75,86]$, and has since been further developed for use in social computing research in situations where sustained, varied access to a sample in the manner of a field site is required, but no actual field site exists, or physical sites do not allow adequate sampling, e.g., studying a phenomenon in a broadly distributed, internally-diverse marginalized community [108].

I employed a Facebook-based ARC with 25 participants, eliciting information on user folk theorization, self-presentation behavior, and adaptation to change over seven weeks of prompts and a closing one-on-one interview. Procedures were approved by my Institutional Review Board.

\subsection{Participants}

While issues around algorithmically-driven systems are essentially universal due to their widespread nature [91], there is good reason to focus this study on a group with heightened self-presentation concerns. Philosophically, this approach flows from feminist standpoint theory, which suggests that members of marginalized communities have the positionality to notice and highlight issues around structures (here, the algorithmically-driven social platforms) that mainstream users might miss $[4,48]$, allowing me to examine both mainstream issues and potentially highly-problematic outlier experiences as well. Past work on how folk theory formation and self-presentation interlock suggests this is the case for self-presentation on social platforms.

Certain groups are more likely to have these algorithmically-related issues on their minds, and therefore it may be easier to elicit necessary details from these groups, which can then be used to guide more general inquiries in future work on broader populations. This is backed up by prior work on the process of identifying algorithmically-driven systems as sources of blame for incidents of failed self-presentation as well, as it relies heavily on expectation violation [35]; it is not unreasonable to assume that those with heightened self-presentation concerns, then, have more detailed expectations and more chance to see them violated. These "heightened cases" of self-presentation have already proved useful in the prior work noted above, e.g. work on Instagram influencers [17], Etsy shop owners [63], and Airbnb hosts [55].

As the heightened case of influencers/business owners has been indirectly covered by prior studies, and I am primarily interested here in the adaptation process of personal, not business, users, I turn instead to the queer community. Self-presentation is particularly salient to queer people due to social stigma. For queer individuals, a lack of deliberate self-presentation decision making can be actively harmful, with consequences ranging from basic exclusion and ostracization to physical harm, especially regarding disclosure of a stigmatized identity [27, 43]. As all members of the queer community, not only influencers and professionals, share this concern, they represent an ideal context of study. This fit is only bolstered by the facts that I am 
a member-researcher, and the version of ARC I employ was developed in the specific context of queer communities [108].

\subsubsection{Recruitment}

As the "site" of research is the queer community, recruitment was focused on constructing a sample that was broad enough to produce transferrable results [45] while also accounting for the internal diversity of the population under study. I used Facebook ads targeted at people 18+ living in the United States who have demonstrated interest in LGBT history, LGBT culture, LGBT community, or LGBT social movements as a primary recruitment tool, supplemented by distribution of recruiting materials through the personal networks of the research team.

To ensure I represented the diversity of the queer community itself, I asked those that responded to my advertisements to self-identify on several dimensions known to be relevant to the diversity of queer experiences, especially in an online context. This included age [19], race [11], gender identity [33, 97], sexual orientation $[108,111]$, and whether a person grew up in and currently lives in an urban, rural, or suburban area [44, 49]. Additionally, I asked if the person had ever been, or aspired to be, a social media professional or influencer. From an initial pool of over 150 respondents, I first excluded past and future influencers/professionals, and then used Trost's nonrepresentative stratified sampling technique [105] to select a group of 41 people to invite which adequately accounted for the factors noted above. Of that group, 35 people consented to participate in the study, and 30 at least completed the opening exercise. Ultimately, 25 people completed the study, a drop-off pattern comparable to past ARCs $[73,74,75,108]$.

\subsubsection{Demographics}

While I have collected demographic data from each participant, I do not include it individually in a participant table, as these characteristics are personally identifying and not the object of study. Instead, I report them here in aggregate.

Participants ranged from 19 to 48 years old $(M=27, S D=7)$. In terms of gender, the sample was $36 \%$ female, $36 \%$ nonbinary, and $28 \%$ male. In terms of sex, the sample was $52 \%$ cisgender and $48 \%$ transgender. In terms of sexual orientation, the sample was $36 \%$ gay/lesbian, $24 \%$ bisexual, $12 \%$ pansexual, $8 \%$ asexual, with the remaining $20 \%$ being otherwise queer. Racially, the sample was $44 \%$ white, $20 \%$ mixed race, $16 \%$ Black, $12 \%$ Latinx, and $4 \%$ each Native and Asian. $52 \%$ of the participants grew up in a rural area; $32 \%$ of participants live in one now.

\subsection{Procedure}

Under normal circumstances, ARCs take place in a secret (non-searchable and private) Facebook group, where participants answer varied weekly prompts directly in the group and engage each other's answers to co-construct knowledge, often building beneficial community in the process $[73,74,75,86,108]$. However, due to concern over participant privacy and how social platforms handle user data, my IRB required us to modify the format into what I refer to as a Protected ARC, which better secures user data while attempting to maintain ARC's community-based benefits.

\subsubsection{ARC Structure and Challenges}

Similar to past ARC studies [73, 74, 75, 108], the Protected ARC was based in a secret Facebook group, with actual data collection via secure Qualtrics forms linked from the group. I chose Facebook as a platform as opposed to other alternatives due to the fact that it remains the 
most used social platform with a private group functionality overall, and also has the broadest demographic reach, lacking any major userbase gaps around important factors such as gender and race [84]. The Facebook group served as an administrative and community space, serving to keep the project's data collection visible to users to prevent drop-off, while also maintaining the direct beneficence of providing a community space for marginalized people, a key benefit of past ARCs [75]. To support both these uses of the Facebook group, I, aided by two research assistants, started and participated in 1-2 community activity threads weekly, separate from the ARC activities.

\subsubsection{Participant Experience}

I ran the ARC during June, July, and August 2019. After initial recruitment per section 3.1.1, which took place via email, participants were emailed a consent form and given the opportunity to ask questions, after which they emailed back a specific consent phrase from the form to indicate their consent to participate. Those that completed the consent procedure and agreed to follow a basic code of conduct were sent an invitation to the secret Facebook group.

Each week for seven weeks, I posted the week's activity and a link to the appropriate Qualtrics form, and answered any related administrative/clarifying questions in the post's attached comment thread. Each week's activity was designed to take a different approach to addressing one or more research questions. Activities ranged from journal writing and shortanswer scenarios to visual elicitation and scavenger hunts and are briefly described and justified in the next section. For full versions of the community (Facebook) and individual (Qualtrics) prompts for each activity, see the Supplemental Materials.

As participants submitted their weekly materials on the Qualtrics forms, an RA and I read the answers and generated follow-up questions, which were sent via either email or private Facebook messages, depending on participant preference.

After seven weeks of prompts, participants were scheduled for individual follow-up interviews with the author. These interviews were effectively a final, summative act of theoretical sampling in the grounded theory tradition [15], acting as an opportunity for broad follow-up on both individual responses and emerging themes. Interviews were text-based and took place on Facebook Messenger, and ranged from 57 minutes to 1 hour and 38 minutes ( $M=74$ minutes, $S D=10$ minutes). All participants sat for an interview.

Participants were paid $\$ 25$ total for their participation in seven weeks of research activities, and an additional $\$ 25$ for completing the exit interview.

\subsubsection{ARC Activities}

In this section, I provide a brief summary of activities and the motivations behind deploying each activity. The first six activities had $100 \%$ participation; the sixth and seventh activities had $96 \%$ and $92 \%$ participation, respectively.

Baseline Self-Presentation Profile: Tell us about your posting to social media - what you post about, why you post it, how you post it, and how you've done this over time. Accompanied by survey items on platforms used and scales. Established each participant's individual profile regarding the context in which they are theorizing, self-presentation, per [25] in order to capture self-presentation style [3], breadth of one's social media ecosystem [27], usage [24], and queer self-presentation-relevant psychometric properties (platform centrality, outness [77], queer identity-related experience [78], self-monitoring ability [70], web skills [52]). 
Lightning Round: Answer three prompts around attitudes and history with platforms, based on common social media activity formats. Would you rather: multiple choice re: posting risk. What's most important: prioritizing platform characteristics. Tag your platforms: make a tag meme in a classic format to assign personas to different platforms you use. Continued building knowledge of individual self-presentation decision making while also investigating attitudes towards platforms, crucial per [23, 80]. Tag meme inspired by past work on folk theories as metaphor [34] and algorithmic personas [114].

Visual Elicitation: Make an image that represents how you think the platform you post to the most gets your posts from you to the people that see them. Elicited partially-latent folk theory information, especially around structure, to access the theorization process from [25]. The visual technique allows flexibility so as not to bias responses [57], is proven in the ARC format [108], and allows spatial benefits of card sorting [25] without limiting participants to the bounds of the research team's existing understanding.

Scenarios: Tell us how you would react to three content posting scenarios: hearing rumors that how content distribution works is changing, noticing something about the posting interface is different, and a posting-related expectation violation incident. Accompanied by need for cognition scale [13]. Elicited change reactions, information gathering, and self-presentation behavior, using scenarios to help increase the salience of the questions while keeping responses grounded in participant experiences $[14,85]$.

Change "Scavenger Hunt": List as many of the changes you've seen on social platforms, big or small, that you've noticed over the last four years as you can, and anything you can remember about how you noticed them. Briefly reflect on which of these might be related to how the platform works, as well as how you feel overall about this history of change. Assessed extent of the impact of change blindness $[99,100]$ while collecting additional data on attitudes towards platforms, using a lightweight format to balance out the heavy writing of the previous and subsequent weeks.

Personal Journal: Tell us about your personal relationship with the platform you use the most, and how this relationship has changed over time, then briefly compare and contrast with your relationships with the other platforms you use. Used contrast journals on differential instances of self-presentation across participant's social media ecosystem [27] to draw comparisons between different platform environments, different attitudes towards platforms, and different behavioral outcomes.

Letter to the CEO: Write a letter to a social media CEO and let them know how you feel about the platform. Then complete a structured "executive summary" which highlights your asks of the $C E O$ and the platform in the areas of content distribution, policy, and interface. Directly elicited user needs while deepening understanding of willingness to adapt, attitudes towards platforms, and regular information foraging routines.

\subsection{Analysis}

My overall approach to this study is based on Charmaz's work on constructivist grounded theory, which puts a premium on being responsive to one's data throughout the research process, including during data gathering [15]. As such, analysis began concurrently with data collection, and continued up through the writing process.

Grounded theory principles significantly shaped data collection, both conceptually and in place. My choice of self-presentation as an embedding context was influenced by Charmaz's advice around gaining context on a larger situation by focusing on a "basic social process" [15 p. 
25]. Initial activity prompts were designed considering the need to establish broad background context around participants, their online settings, and their processes, key for informing later theoretical sampling as well as assessing transferability [15, 45].

Each of the two RAs maintained weekly trend memos, which aimed to look across all the data for each week and develop per-activity patterns, while I maintained a set of memos linking these emerging trends across weeks, based on her own observation and reading/discussing the RA memos. The interplay between author and RA analysis was particularly helpful in actively balancing structural and affective concerns in analysis. This memoing was followed by discussion among the research team to compare analysis, put that week's emergent memos in concert with past weeks, and craft quick follow-up questions.

I then used these memos and follow-up question responses to adjust activities in subsequent weeks, in order to be responsive to emergent themes. For example, by the time the team analyzed the week five prompt data, it had become clear that overall relationship with platform was a major factor in how people were making adaptation decisions. As such, I adjusted the week seven prompt, allowing me to continue developing the emergent concepts which now inform section 4.3 .

At the end of the seven weeks of prompts, all data and memos were rapidly re-analyzed and discussed among the research team in order to create individualized interview guides which covered emergent themes in the specific context of each participant's past responses. This allowed the interviews to be guided by emerging theories, and served as a lightweight form of member-checking, similar to [108].

After data collection, the study team engaged in multiple rounds of coding, memoing, and discussion to further refine our analysis, using MAXQDA as our primary analysis tool. This included a full open coding round and focused coding informed by author and RA memos, with additional open coding as dictated by focused coding findings. Finally, I conducted a round of theoretical coding and integration, an advanced coding step in which focused codes are related to each other and, if appropriate, to existing theory [15].

One of the primary benefits of using an ARC approach is that it not only allows, but effectively demands triangulation of data from multiple sources, leading to a more robust and holistic understanding of the phenomena under study [73, 74, 75, 86, 108]. This was true throughout my analysis. All results reported below are based on triangulation across multiple ARC activities unless otherwise noted.

\subsubsection{Positionality}

All interpretation is informed by the positionality of the research team relative to the participants and subject matter [15]. I am a member-researcher in the queer population under study. Specifically, I am a transgender woman and a lesbian. The rest of the study team included both cisgender and transgender women, and team members who were both cisgender and heterosexual completed a training module on working with LGBTQ+ populations.

One important limitation to this work is directly tied to the positionality of the research team: I do not thoroughly account for the impact of race in this study. While this was not the primary focus of the study, it is important to be mindful that I am white, as are two of the three research assistants (the third being East Asian). This could have resulted in underestimating or simply not being cognizant of racial impacts, and I specifically call for future work that investigates the impacts of race, especially as they concern attitudes towards platforms as discussed in section 4.3 . 


\section{RESULTS}

I find that everyday adaptation to changing algorithmically-driven social platforms appears to be a process that has variable outcomes based on the level at which one is able to consider and construct useful folk theories, the extent to which both the change and platform in question honor the user's conception of platform spirit, and the extent to which users are attached to/embedded within a platform. In the following sections, I will first lay out a system for classifying folk theorization (RQ1), and then use this system to highlight important cleavages and opportunities regarding noticing change (RQ2), choosing to adapt (RQ3), and the act of adaptation itself (RQ4). These results are summarized in the form of model adaptation pathways in Table 1.

\subsection{Classifying Folk Theorization: Theorization Complexity Level}

RQ1 asked if and how users are actively theorizing about algorithmically-driven social platforms. All participants had at least basic awareness of the fact that algorithmic mechanisms were at play on modern social platforms, but beyond this the level at which the participants were theorizing varied. As this project focuses on a process of adaptive reaction to change, I will concentrate here on how theories are being formed and updated, and not the theories themselves.

Ultimately, my inductive analysis suggested four distinct levels of folk theorization complexity: basic awareness, causal powers, mechanistic fragments, and mechanistic ordering. Comparing across these groups in light of prior work in folk theorization and literacy (e.g., [29, $60,61]$ ), a classification system with two broad categories emerged. I call this the individual's Theorization Complexity Level (TCL), defined as the level of system complexity a user is aware of, takes into account, and employs to pursue their own goals when folk theorizing. Each TCL is additive, carrying the characteristics of prior levels in the hierarchy. As users move up this classification hierarchy, the way in which they are conceptualizing of algorithmic systems becomes more complex and involves more information.

\subsubsection{Functional Theorists: Basic Awareness and Causal Powers}

Functional folk theorists have folk theories that reflect that they are focused on the presence and effects of algorithmically-driven systems, as opposed to the causes, or inner workings of the system. The types of theories generated by functional theorists include what DeVito et al. referred to as "abstract theories," [26] and the functional theorist's process reflects the associated "algorithm... as an other or interloper." There are two distinct levels of functional theorization: basic awareness and causal powers.

Folk theorization can be classified at the level of basic awareness if the theorizer indicates that an algorithmic/computational system is in play on a platform, having some effect, but does not assert or reflect knowledge of a specific effect. The algorithm, essentially, is doing something in the view of a functional theorist, but as P13's visual exercise (Figure 2a) demonstrates, exactly what it is doing remains a mystery (or, to P13, "internet magic").

Moving up one level, folk theorization can be classified at the level of causal powers if the theorizer indicates that an algorithmic/computational system plays a causal role in a distinct outcome/outcomes. While a basic awareness asserts some effect, causal powers are distinct in that they assert a specific effect. P35's map (Figure 2b) demonstrates this via direct contrast with P13's basic awareness. While P13's theory has a platform's mechanisms in a nonspecific role in the building of a feed one's friend would see with no indication of curation, P35 visually 
indicates (and follows up in their interview to confirm) that they see a platform mechanism as using some criteria to select which posts friends are shown and in what order.
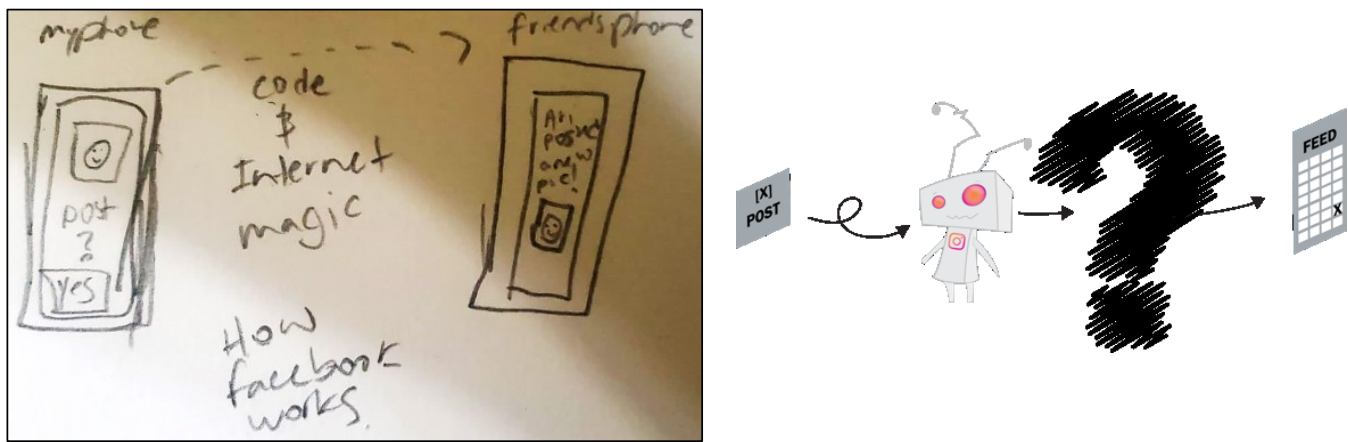

Figure 2: A) P13's visual exercise demonstrating characteristics of basic awareness, a type of functional folk theorization. B) P35's visual exercise which recognizes the impact of an algorithmic system (here, presence and position of content in a feed), but not any structural detail, characteristics of a causal powers level of folk theorization. (Participant-submitted images, sharpened for clarity. Larger versions of all visual exercises shown here can be found in the supplemental materials.)

\subsubsection{Structural Theorists: Mechanistic Fragments and Ordering}

Structural theorists dive into the causes behind the algorithmic/computational effects they encounter. These users often confidently assert effects - in the present study, structural theorists universally took algorithmic curation as given and focused on the "how" of the curation. Theories generated by structural theorists fall into the "operational theories" distinction proposed by DeVito et al. [26], and encompass many of the folk theories found in prior work (e.g. [25, 31, 34, 88, 89]). There are two distinct levels of structural theorization: mechanistic fragments and mechanistic ordering.

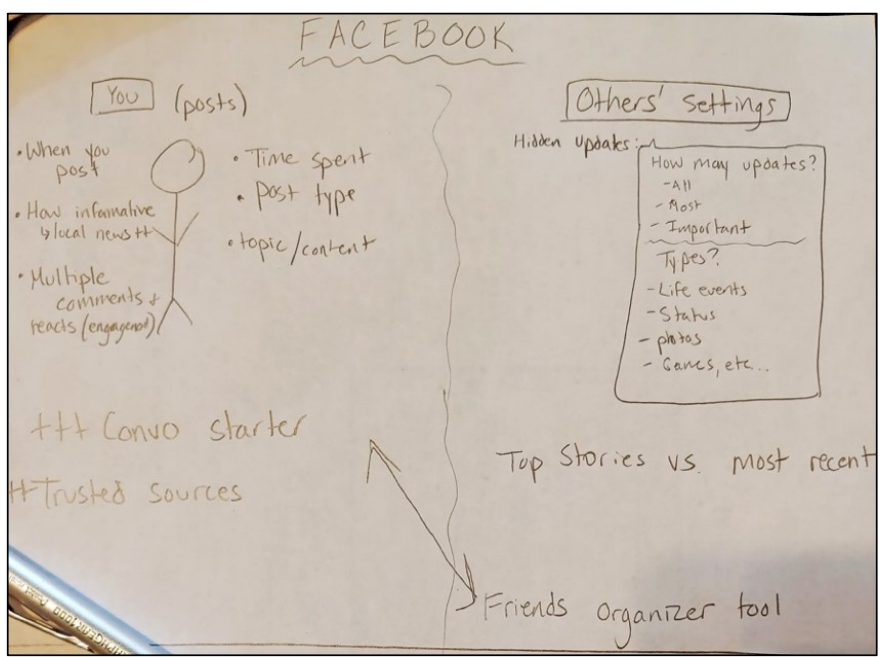

Figure 3: P19's visual exercise showing multiple factors which may impact content curation, indicating structural mechanistic fragments folk theorization. (Participant-submitted image, sharpened for clarity.) 
Folk theorization can be classified at the level of mechanistic fragments when the theorist indicates that an algorithmic/computational system plays a specific causal role (or roles) on a platform, and believes that they have identified multiple specific factors/datapoints that are weighed by the system in some fashion to make decisions. The participants who theorized at the level of mechanistic fragments have a mental list of possible criteria for algorithmic decision making, which they then regularly shuffle, re-weight, subtract, and add to when puzzling out how platforms work. As P19's cognitive map (Figure 3) demonstrates, there are often internal gradations regarding the weighting of criteria - in P19's case, a distinction between the yourelated and other-related factors that you/other have direct control over (comment engagement, content type, etc.), and those that can either override algorithmic mechanisms (the top stories/most recent toggle) or those that get extra weight and require demonstrated behavior over time (being a conversation starter or a trusted source).

Finally, at the top of the hierarchy, folk theorization can be classified at the level of mechanistic ordering when the theorist indicates that an algorithmic/computational system plays a specific causal role (or roles) on a platform, and believes they have identified not only multiple specific factors/datapoints used to make decisions, but also the causal pathways within this set of factors in the form of either complex rankings/weightings or literal assertion of decisionmaking pathways. This is the next step up from mechanistic fragments - as P1's map (Figure 4) shows us, the mechanistic fragments are now arranged in a way that not only gives the theorist a working theory of what criteria might be important to a platform, but also when, how, and where that criteria comes into play in actual decision-making.

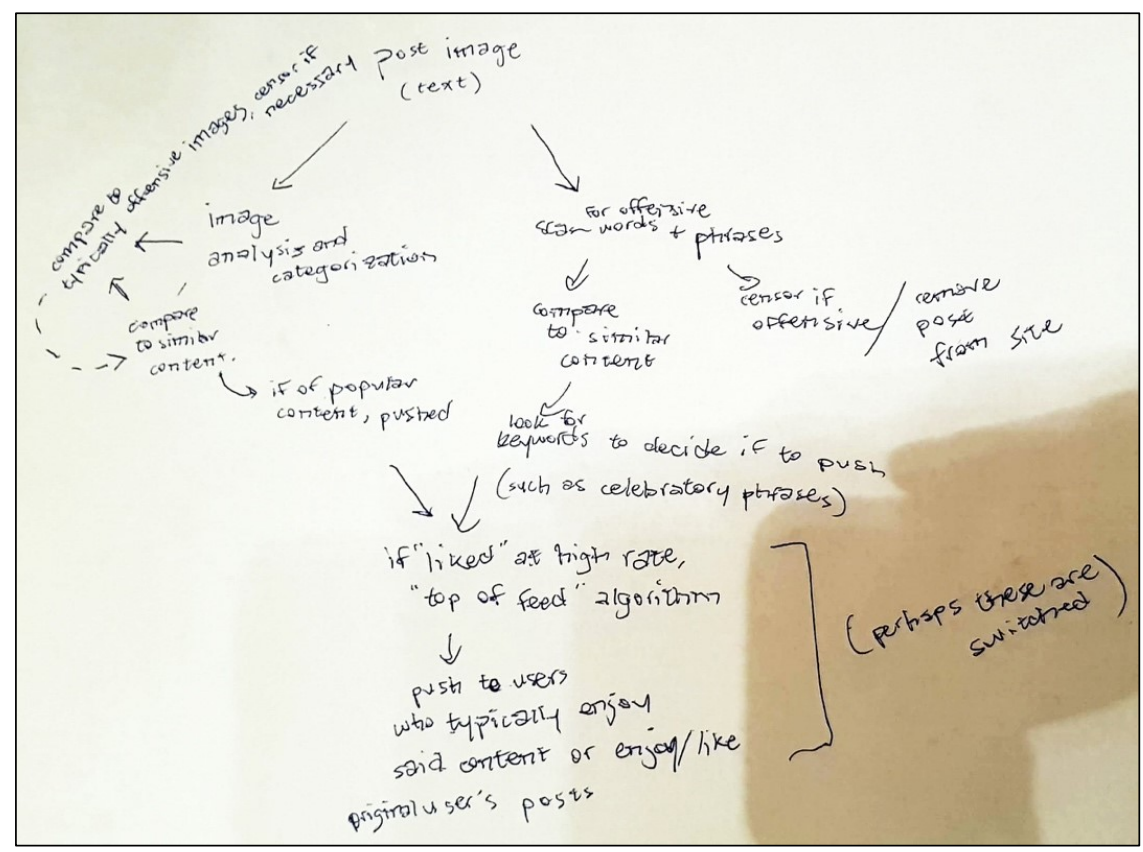

Figure 4: P1's visual exercise, showing not only multiple criteria but specific causal pathways, indicating structural mechanistic ordering folk theorization. (Participant-submitted image, sharpened for clarity.) 
As is the case with P1, these understandings are often expressed through if/then/else logic (e.g., P1's IF liked at high rate THEN post to top of feed ELSE post to niche audience based on other criteria), and often contain references to additional computational processes assumed to be in play (e.g., P1's assertion of an "image analysis and categorization" function).

Theorization at the level of mechanistic ordering does not require what Keil calls "full mechanistic details." While some users theorizing at this level do certainly have the "mental blueprint" characteristic of this kind of causal pattern knowledge [61], this is not necessary, as full mechanistic detail is not only rare as it is in folk theorization generally, but also perhaps impossible due to both rapid system change and the deliberately-opaque nature of platforms $[38,82]$.

\subsection{Noticing Change: Change Types and Tipoffs}

RQ2 asked about how users notice change. Overall, the data suggests that users do notice change on algorithmically-driven social platforms. Users are not unaware of the constant updates that surround them; none of our participants failed to notice change entirely. A minority of participants insisted they had seen no change, and then proceeded to extensively describe changes (and had described changes prior to saying there were none), suggesting that these participants actually having a working awareness of change. Participants noticed two types of technological changes: feature change and algorithmic change. Within each TCL, there seem to be differences in amount of change noticed as well as what factors are cueing the user into the fact change has occurred, which I refer to as tipoffs, and which correspond to the information sources in the current model of folk theory formation (Figure 1, top right).

\subsubsection{Feature Change}

A feature change is a change asserted by the participant which concerns the platform's interface (including aesthetic and organizational changes) or a feature of a platform (e.g., a privacy control). This type of change deals mostly with changes to visible options or presentation, not to behind-the-scenes processes. Changes of this type can range from P19's noticing of new location-based options and bitmoji on Snapchat and P2's excitement over Polls being added to Facebook Groups, to P8's frustration over what they perceive as a constantlychanging basic layout on YouTube and P17's casual realization that they could keep typing after 140 characters on Twitter.

Every participant talked about at least one feature change, which appears to be the most noticeable type of change overall. Feature changes represent roughly half the changes reported by high and functional theorists. However, these groups are tipped off to feature changes differently. Structural theorists frequently notice feature change by direct endogenous use of new functionalities, and are additionally prompted by exogenous information (e.g., hearing from friends). By contrast, functional theorists seem to rely on endogenous information primarily and generally notice feature changes via visual interface elements, suggesting that functional theorists may generally benefit from changes being deliberately linked to visual alterations.

\subsubsection{Algorithmic Change}

An algorithmic change is a change asserted by the participant which concerns how one of the algorithmic systems at work on the platform operates. Importantly, it is not necessary that the participant explicitly recognize or label this as an algorithmic change; I count a noticed change 
as algorithmic when the participant talks about an automatic system process that involves user data or its effects, regardless of if the participant is aware of the fact that they are talking about an algorithm. This leads me to break algorithmic change down into explicit and implicit subtypes. Explicit algorithmic change is noted as being related to an algorithm/algorithmic system directly, though this awareness of an algorithm need not be specific to a single algorithm, or even the details of the algorithmic process. Rather, this distinction captures participants who notice change and assume that it is tied to an algorithmic process, such as P25:

“Algorithm must have changed or my friends don't get on as much? I notice I don't get nearly as many reacts, comments, shares as I used to 5 years+ ago. I notice even when I follow a page, if I don't react, comment or share something from it every so often, I start seeing less and less of it as if FB assumes I want to see less because I'm not interacting with it as much."

While P25 clearly has a limited understanding of what algorithm is changed, and even exactly what those changes are, they do have enough knowledge to identify the algorithm as the likely site of change. Essentially, they know where to look should they want to try and address this change. However, explicit algorithmic change only accounts for about a third of the algorithmic change in this dataset; implicit algorithmic change is noticed at roughly double the rate of algorithmic change. Implicit algorithmic change is noticed change which, from a technical perspective, is clearly a change to an algorithmic component of a platform, but which is not specifically called out as such by the participant. This reflects tacit knowledge of the presence of an algorithmic system: the participant may not be able to use this language, but they are clearly aware that a system we know to be automatic is the site of the change in question. Frequently, participants conceptualize these changes in the language of "automatics" or the assertion that some decision making force is assembling content feeds in a certain, now changed, way, e.g., P29's new awareness that post time was no longer a good predictor of who would see their tweets:

"On Twitter, it's now possible that people are seeing my tweets at the top of their timelines even if I posted the tweet a while ago due to the 'In case you missed it' feature."

While P29 does not specifically say that there is an algorithmic system at play, they are clearly noticing a change to an algorithmic system, and therefore essentially have a direction to move in should they wish to investigate the change, though certainly a less clear direction than one who explicitly notices algorithmic change.

Overall, all but three participants noticed some type of algorithmic change. While both functional and structural theorists recognize a robust amount of algorithmic change, structural theorists seem to more readily recognize these changes as explicitly algorithmic.

While endogenous tipoffs continue to be most common here, structural theorizers tend to notice more algorithmic changes via talking to friends and reading the news than other groups. Within endogenous information, there are also differences in focus across different TCLs. Functional theorists tend to notice algorithmic change via content-related tipoffs, e.g. changes in content distribution patterns (especially around perceived increased personalization) and what content is being rejected/moderated. Structural theorists tend to notice more via engagement- and network-related tipoffs, e.g. changes in the level of engagement they see on their posts. 


\subsubsection{Barriers to Noticing Change}

Though the participants were largely aware of change, and have had some success overall identifying specific changes, it is unlikely that all possible changes were noticed. Additionally, three participants $(3,16$, and 31) did not notice algorithmic change specifically. Clearly, there are still barriers in place to noticing change. One of these barriers may be an issue of change type conflation. At a broad level, the data suggests that users may be "getting their wires crossed" when it comes to noticing different types of change, likely due to the fact that some kinds of change are simply more salient and easier to notice for the average user. At multiple points in the study, but especially in week four's scenario exercise and week five's diary exercise, I prompted users to recount specific types of changes. While users did notice all types of changes, they often bucketed them in a way that suggests that many changes, of all types, are understood as feature changes. When asked about change generally, feature changes were by far the most common type described; when asked about feature change specifically, most changes listed were feature change, but a notable minority were actually implicit algorithmic changes. When asked about algorithmic change specifically, slightly under half the changes listed were in fact feature change, outstripping explicit algorithmic change. One possible explanation is that feature change is simply more salient overall due to its upfront and often large-scale and visual nature, compared to the potentially change-blindness-inducing nature of algorithmic change [99, 100], and therefore may "crowd out" algorithmic change in some attribution cases.

\subsection{Platform Spirit and the Adaptation Decision: Adapt, Limit, or Leave}

RQ3 asked if and how users choose to adapt to platform change. I find that users largely see change as labor, and make a decision about if they should put the labor in to adapt, or simply limit their participation or leave a platform largely based on if they see the change and, more broadly, the platform's current behavior, as honoring or violating their perception of the platform's spirit.

Importantly, during the grounded theory inquiry, it became clear that in a social platform context, spirit and task-technology fit require reconsideration. Specifically, users constantly conflated the two concepts, with lack of task-technology fit often viewed as a violation of spirit. As I have committed to center this study in user perception, from this point on I use the term platform spirit, which I define as the user's perception of what a platform is and what it is for, as determined by the user's understanding of the platform's stated mission, its values and actions in practice over time, and the functionality which it allows as juxtaposed with the user's understanding of the platform's purpose.

Overwhelmingly in this sample, if a platform is perceived as being faithful to a user's understanding of the platform's spirit, they will attempt to adapt. As I will discuss in the next section, they may fail at this adaptation, but they will generally make some kind of attempt. If the platform is perceived as being unfaithful, however, different types of choices are made across different levels of folk theorization. In order to better illustrate these differences in how platform spirit appears to impact the foraging -> sensemaking -> theory side of the folk theorization loop (Figure 1, left side), I will follow one exemplar case through the decisionmaking process for each level. 


\subsubsection{Adaptation as Labor}

Across the board, participants recognized adaptation as a form of labor they are effectively being asked to do to successfully continue their use of the platform. This labor and the associated user concerns stem from not only the work of adaptation, but the conditions under which adaptation happens. For example, the unstable nature of platforms and constant need to adapt is a large amount of labor for many, leading to an expressed sense of exasperation and some level of jadedness about if this effort will last. P8 expressed this clearly: when asked during the week four prompt how they react to news change is coming, they said "My immediate gut reaction is 'oh no, not again," which they then further explained in the followup interview:

"It's usually a feeling of needing to adapt to something that is most likely going to be obsolete in a short time later. Sometimes the change is done so unannounced that it's also a mix of 'great, now I need to learn how to navigate this site I had already learned a lot of ins and outs of all over again."”

P8 has already sunk time into learning a platform and is not excited to contribute more of their time just to learn to keep doing what they are already doing. P20 said they feel similarly, and even have stricter standards than P8: changes get an hour of attempting to adapt, maximum, as they feel that their "time is valuable" and anything past an hour is not a good enough use of their time.

This frustration regarding being asked to do adaptation labor is sometimes also intensified by the fact that not all users accept all changes as necessary, and some even prove to be counterproductive, leading to frustration over the need to adapt to unnecessary changes, as P16 expressed:

"At the risk of sounding like an eighty year old grandma, there is far too much change on this site. I do generally believe that updates are good. Changing major features every three weeks, however, is not ..... too much change leads to overcomplicating very straightforward tasks. Please keep in mind that the wheel does not need to be reinvented"

Even for those participants who initially desire to adapt, the fact that this is labor eventually catches up to them. P13, for example, reported a pattern of trying to adapt turning into complaining and eventually into losing interest due to the frequency and magnitude of change. P35 reported a similar pattern, summing things up well:

"...constant change makes me check it out at first but then I get tired of the changes that happen so often that I decrease my presence."

The fact that users see adaptation as labor sets up a choice to put this labor in or not - a choice that appears to be largely based on what a change does to a user's perception of their relationship with the platform as they understand it.

\subsubsection{Functional Theorizers}

In this sample, functional theorizers attempt to faithfully adapt if a change is faithful to perceived platform spirit, and tend to choose not to put labor in and adapt if changes violate perceived platform spirit. If the platform in question is not an essential part of the user's life, they generally leave entirely. If a platform is crucial, they heavily limit their participation. For 
example, P15 is working at a causal powers level of folk theorization, knowing that some part of each platform picks what one will see on a feed, but not how or what factors will influence this curation. When P15 encounters a change that is faithful to platform spirit, they choose to attempt to adapt - in fact, so long as changes improve the platform, are not overwhelmingly frequent, and are well-communicated, they're quite accepting of and game to try and adapt to change. P15 notes:

"It's not that social media platforms shouldn't innovate; they should innovate consistently and not often so that users get the best experience and still feel comfortable posting and sharing."

However, some major platforms have made changes that bother P15. Some directly violate the core use case $\mathrm{P} 15$ has for the platform, e.g. Tumblr:

"Tumblr has honestly always been the site for sexuality, sexual freedom, and sexual expression, that is, until its recent ban on pornography, nudity, and female-presenting nipples. I know that many people, not just LGBT folk turned to this platform to figure themselves out or to connect with others with their interests."

P15 was one of the people trying to use the platform to connect with others and perform important identity exploration, and Tumblr introducing new algorithmic curation standards around content crucial to the LGBTQ+ community made that almost impossible for P15, who ultimately left Tumblr entirely instead of trying to adapt. There was no point to adaptation the core use case for the platform was gone.

P15 has slightly different concerns with Facebook's repeated changes, noting that Facebook has shifted away from what P15 sees as their core purpose:

"It seems to have lost touch with its user-base and its mission, to connect people."

More specifically, P15 sees changes that increase the amount of curation and targeting on the platform, which violate both P15's understanding of what purpose Facebook is meant to serve (as changes have made it "a lot less user-focused and more revenue-focused") and their understanding of what place Facebook fills in their life and in their self-presentation activities as a person with a heavily authentic self-presentation style:

"I began to notice my feed being more "curated" than I really wanted and began to limit my use"

This increased curation made P15's use cases around sharing interests more difficult, and also requires more labor in terms of playing to the algorithm with one's content, which conflicts with P15's authentic self-presentation style. P15 noted they would have liked to leave Facebook entirely after these unwanted changes, but settled on a strategy of heavily limiting their participation because they recognize Facebook as an essential platform with so many crucial connections that they can not leave without difficulty. As they noted, unhappily:

"The platform everyone hates but everyone uses. You have it because of its prevalence, not because you want it." 


\subsubsection{Structural Theorizers}

Structural theorizers in this sample faithfully adapt if a change respects the platform's perceived spirit, and bifurcate into two subgroups when changes violate perceived platform spirit - one group tends to limit and leave, while another subgroup stays and adapts.

P31 theorizes at the mechanistic fragments level, and when they encounter changes that are faithful to platform spirit, they easily adapt. They are not always happy about adapting and have many negative things to say about platforms they've adapted to, but ultimately, so long as platform spirit is honored, they find a way. For example, P31 sees constant, haphazard updates on Tumblr which seem to not reflect user input, characterizing the platform as "disorganized at best but more often chaotic." And yet, despite frequent annoyance, P31 adapts to changes from what is now their most crucial platform. P31 sees Tumblr as having a very distinct purpose:

"It's meant to be used to connect people and create community while also being able to declare who you are (or at the very least, give a safe space for exploration and discovery)."

Despite being annoying, Tumblr still fulfills what P31 sees as their core purpose, not making changes that, in P31's opinion, damage that sense of safety. This fulfills Tumblr's perceived commitment to the community and also enables P31's personal efforts to educate on social justice, which require such a safe space. This is to the extent that P31 says they "don't feel as safe posting more personal feelings or thoughts on other platforms.” As such, P31 adapts. Notably, P31 also said it is helpful that Tumblr clearly acknowledges issues with their changes a direct contrast to other platforms such as Facebook.

There is much P31 liked about Facebook: they view it as consistent, easy to use, and full of "a great number of features that help people." It has broad audiences, and could potentially be an excellent place to educate. However, P31 now sees Facebook as engaged in what they perceive as a large-scale spirit violation enacted through Facebook's moderation systems:

"It is more likely for individuals from marginalized groups to be suspended when they defend their right to not be harassed for their very existence. My safety in using Facebook would increase if Facebook took a stand against individuals that promote hate speech against protected groups."

For P31, Facebook is not living up to its responsibilities as a platform, and either changes to the moderation systems or a lack of appropriate changes to the moderation systems has made it a particularly unsafe space for marginalized people. Notably, they also do not do enough to address why the platform behaves this way, P31 notes:

"It undermines my confidence in the platform as a whole if they aren't transparent with something so vital to the safety of their users."

As a result, P31 has severely "curtailed" their posting to Facebook.

A subgroup of structural folk theorists seems to behave differently than the rest when faced with platform spirit violations. For example, P21 theorizes at a mechanistic fragments level, and behaves similarly to P31 when faced with a change that is faithful to platform spirit. This is true even in cases where changes are confusing and inconsistent. However, as a member of this subgroup, when faced with changes unfaithful to a platform's perceived spirit, P21 does not limit or leave - rather, they adapt. For example, P21 considers Facebook to be a crucial platform for connecting with people who actually know them, describing it as their "most social social 
platform." However, P21 believes that a change to Facebook's curation systems represented a direct threat to their social use case:

"When Facebook said it was going to choose FOR ME who out of my friends were worthy to be seen on my timeline, I tried to use that against it."

P21, despite encountering the type of use case violation which causes most to either leave the platform or limit their participation, stayed and adapted - specifically, via gaming the system, which I will return to in the next section when discussing unfaithful adaptation.

These unfaithful adaptors appear to share a distinct set of traits when compared to other structural theorizers that do not engage in unfaithful adaptation behavior. They generally rely more on endogenous information for theorization, especially direct, sustained observation of platforms. When they do rely on exogenous information, it is from news articles, whereas structural adaptors in general usually have a broader base including community members. They also expressed more opinions about platforms, both negative and positive. In essence, this appears to be a group of users who are closer to the platform, both in terms of caring about it and feeling a sense of ownership, as well as ability to adapt simply by studying the platform.

\subsection{Adaptation: Foraging, Faithfulness, and Resistance}

RQ4 asked how users actually adapt, both informationally and behaviorally - essentially, how the folk theorization loop in Figure 1 plays out in the new context of change. This seems to vary by TCL, with functional theorizers tending to make small adjustments based on low levels of information and structural theorizers often making both faithful and unfaithful adaptations based on expanded information networks and experimental behavior. What appears universal in this sample is where behavioral change starts: I saw no instances of radical new behaviors being introduced, but rather various (and variously successful) gradations of adjusting or otherwise updating one's existing self-presentation tactics and adopting tactics similar to one's own existing repertoire.

\subsubsection{Functional Theorizers}

When participants theorizing about platforms at a functional level attempt to adapt, the result seems to be a small amount of additional information foraging and small adjustments to existing self-presentation tactics. Functional theorizers are not so much adapting to the algorithmic systems on a platform, but rather adjusting around the algorithmic systems by using limited knowledge to tweak ways of behavior that have worked for them previously. They are also doing so under stress, and with no guarantee of success.

The foraging step for functional theorizers is best characterized as limited. Commonly, participants at this level took a watch and wait approach, increasing their endogenous information via observation of the platform. For some participants, such as P34, this includes temporary limiting of participation:

"I might avoid posting for a while until I understand better."

Others engaged in minor, very scoped on-platform testing behavior, but were hesitant. P20, for example, "plays around" with posts only after some time has passed since they noticed the change, after they have had the chance to watch and wait and make sure their playing around will not immediately lead to negative consequences. This testing behavior is often quite scoped; P20 and P8, for example, put time limits on their testing and exploration, as P8 explains: 
"I mainly try to spend an hour or so of my free time to tackle navigating the new updates, mainly to figure out how to find content or information that I regularly engaged with prior to the update."

This direct endogenous testing and observation, light as it may be, appears to be crucial for functional theorists. However, as P8 continues, exogenous sources become useful in difficult-toparse change situations:

"I mostly learn better through discovery on my own experiences, and I usually seek others help when I'm struggling with finding things"

This pattern of gathering minimal additional information by slightly expanding one's usual sources and testing behavior seems to be typical with functional theorizers and can run both ways. P35, for example, consults friends in online groups and then does some minimal onplatform testing to verify what their friends have told them. Functional theorizers are approaching change with more knowledge than they had before - but only a small amount more.

Once they have foraged, functional theorizers appear move on to actual behavioral adaptation, but notably do so with a distinct sense of resignation. For example, $\mathrm{P} 4$ refers to the "continuous cycle of updates" as "background noise" - just a fact they will have to deal with. P8, while recounting their basic adaptation strategy, illustrates the general attitude well:

"I would initially be confused. I'd look at what changed, see what new options are available and which ones are gone and maybe test them out as I post. Alternatively, I would just try to trudge along as normal because sometimes social media can't keep the same interface for a year."

P8 not only captures the attitude, but an important point about functional theorists generally: adaptation is not guaranteed. Sometimes functional theorizers do just have to trudge along or else eventually limit their participation or leave, even if they have made the decision to try to adapt. P34, for example, does very little to adapt, very lightly attempting to tweak their content. A combination of low ability to theorize in order to adapt and an authentic presentation style that is damaged by going much further than they already do with their go-to content-related self-presentation tactics leaves P34 in a position where they usually try to adapt, but ultimately fail and heavily limit their participation.

For those that do manage to adapt, the focus is not actually on adapting directly to the algorithm. Only one participant actively spoke in terms of adapting to an algorithm specifically; the rest of the functional theorizers talked in terms of features (e.g., P20), options (e.g., P34), and privacy settings (e.g., P35). While all of these relate to the algorithmic system, and can be read as a proxy for it, it was clear that participants at this level were not conceptualizing the algorithm specifically as what needs to be accounted for.

Instead of focusing on understanding and accounting for the algorithm directly, functional theorizers appear to be thinking in terms of adjusting traditional self-presentation tactics. For example, P13 heavily leans on privacy settings as a self-presentation tactic and has adopted a practice of regularly checking and tweaking these controls to deal with change on a per-post basis. Similarly, P8 is used to using content-based strategies where they adjust the content of their posts to manage their self-presentation. They adapted to changes that reduced their perceived audience via cycling though new content types, temporarily trying to just repost, and posting more frequently in an effort to be more appealing. Ultimately, the adaptation behavior 
observed for functional theorizers is best characterized as lightly tweaking existing behavior based on a small amount of new information, with failure as a possibility.

\subsubsection{Structural Theorizers}

Participants theorizing about platforms at a structural level appear to adapt in two distinct patterns. As noted earlier, a subgroup of structural theorizers is the only group of participants in this sample to actively try adapting instead of limiting or leaving in a situation involving platform spirit violations. However, all structural theorizers seem to be adapting directly to algorithmic issues by making substantial adjustments to their existing self-presentation tactics, including reprioritization of those tactics. They are largely successful at these adaptation attempts.

When foraging for information to respond to change, both types of structural theorizers undertake expansion of their sources to more of an extent than functional theorizers, and do so across both endogenous and exogenous sources. The difference lies in what sources are ultimately favored during this expansion. Faithful adaptors tend to favor exogenous information or stay balanced between endogenous and exogenous. For example, to help adapt, P9 expands their information sources from personal sources (e.g., talking to/asking friends) to also include official sources such as platform help documentation. P10, meanwhile, steadily ramps up the volume of their foraging across both source types, as do P12 and P19, while P31 not only accelerates both types of foraging, but then explicitly starts to use their expanded experimentation as a tool to verify information from their expanded exogenous sources.

By contrast, unfaithful adaptors tend to double down on endogenous information. This includes increasing direct observation of the platform, but it more heavily leans on experimentation-type behavior across the board. This is not to say that faithful adaptors at this TCL do not experiment - they do, at a higher level quite distinct from the small-scale testing seen in functional theorizers. However, unfaithful adaptors go beyond this, with more and more elaborate experimentation that may involve multiple people. For example, P2 recruited friends for their experiment to test out their folk theory that different reactions on Facebook (e.g., like, love, anger, laughter, etc.) had different impacts on content distribution:

"I got 9 friends to join me in an experiment. We selected 5 outside our clique of 10 friends, 5 pages we commonly liked and 5 groups we all shared. Three of us would only like content from all 3 categories. 3 of us would only love content, 3 of us would react as natural from all emotional options, and 1 would not react at all. We charted how often the content from the designated pages, groups and friends came up in our feed, and found that the loving content positively increased frequency on newsfeeds."

While not all experimentation by unfaithful adaptors is this elaborate, in this sample only unfaithful adaptors went to these lengths, and it seems that only unfaithful adaptors approach such experimentation with an overall attitude of excitement.

When it comes to actual adaptation behavior, the faithful and unfaithful appear to more thoroughly diverge, but the core difference between structural and functional theorists seems to be that structural theorists are adapting in ways that directly address what they think is happening algorithmically on a platform. For example, P12 theorizes at the level of mechanistic fragments, believing in several different factors which could impact algorithmic decisionmaking. This includes network analysis as "some way of connecting me to other people like me on social media." When P12 encountered a change in content distribution that made it seem like 
celebrity and influencer status was a crucial factor in decision-making, P12 began adjusting their own network to try and clearly communicate to the platform that they were not like and did not want to see or be grouped like influencers. This is a response directly to algorithmic change (here, a switch to a non-chronological feed suddenly boosting influencers) which rearranges components of P12's existing folk theory in order to inform an adaptation strategy, typical of structural theorizers.

While this direct reaction to the algorithm usually results in adaptations specific to the perceived algorithmic change, and both faithful and unfaithful adaptors instantiate these adaptations as extensions of existing self-presentation tactics when platform spirit is being honored, unfaithful adaptors seem to behave differently when faced with situations that violate platform spirit. These participants take their folk theory and, effectively, fight back against the platform. For example, P21, who sees being able to connect with the entire community they have built on Facebook as a core part of the platform's spirit, believed that Facebook had switched to prioritizing content distribution between one's most interacted-with friends, a violation of that perceived spirit. In response, P21 started searching for a wide variety of friends, including those they did not frequently interact with, and liking many of their past posts essentially sending counter-signals to the algorithm to counteract the new curation style. Others adapted in thematically similar but situationally unique ways: P1 saw undue boosting of influencer content and started to use their account in an influenceresque "spam" style as a counter. P7 went even further, asking their audience to directly comment on posts to help counter a perceived change towards favoring content with heavy interaction, which violated P7's education and activism-focused use case.

\subsubsection{Barriers to Adaptation}

The adaptation pathways described above point to barriers to adaptation generally, especially for functional theorizers, who largely appear to be held up by inadequate baseline understanding of the platform to work from. However, I also directly asked participants what would make things easier for them during the week seven activity and regularly asked followup questions around what would have been helpful in actual adaptation scenarios participants described. While some barriers (e.g., lack of adequate advance notice of change leading to lack of time to rapidly adapt) were universal, I also saw variance across TCLs, suggesting a need to carefully tailor interventions based on the level on which a user is already thinking about a platform. For example, both functional and structural theorizers see lack of information as a barrier; however, why they see it as a barrier varies. Functional theorizers appear more concerned with the complexity of information, as P13 notes:

"It would be nice if it was written in easy to understand language and not a large wall of text."

For functional theorizers, information that is too complex and detailed seems to be both overwhelming and discouraging. By contrast, structural theorizers do not seem to see complex information as a barrier, but are instead more concerned with the organization of this information, including how easy it is to find relevant, and avoid irrelevant, information, as P10 describes:

“...a forum or location being dedicated specifically to updates and changes. A few places have 'help and info centers' but they're often kind of difficult to maneuver so it would be nice to have better search functionality and a 'TLDR' section that sums up 
the really complex info that might pertain to flowery legal jargon etc.... the main thing now is that a lot of [adaptation] is speculative or trial and error. It would be great if it were explicit."

For the structural theorizer, the barrier is a lack of efficient ways to find and navigate the complex information they have no trouble integrating into their theories. Additionally, structural theorizers pointed out one specific piece of missing information that they find makes it more difficult to adapt: the "why" behind the changes.

\section{DISCUSSION}

In this study, I have contributed a new system for classifying user folk theorization in the context of social platforms, as well as a set of adaptation pathways, for the context of selfpresentation via said platforms, summarized in Table 1 . While each user's ability and path to adaptation will to some extent be unique, better understanding these adaptation pathways in the context of how the user is theorizing about the platform gives insight into both the process of folk theorization and how it impacts self-presentation. In this section, I will cover the implications of this study for algorithmic literacy, for platforms, and for the LGBTQ+ community, but will first briefly review three contributions to ongoing work on folk theorization and self-presentation: the establishment of an interplay between sensemaking and foraging, the integration of folk theorization complexity into our models of both processes, and a revised concept of platform spirit and how it impacts both processes.

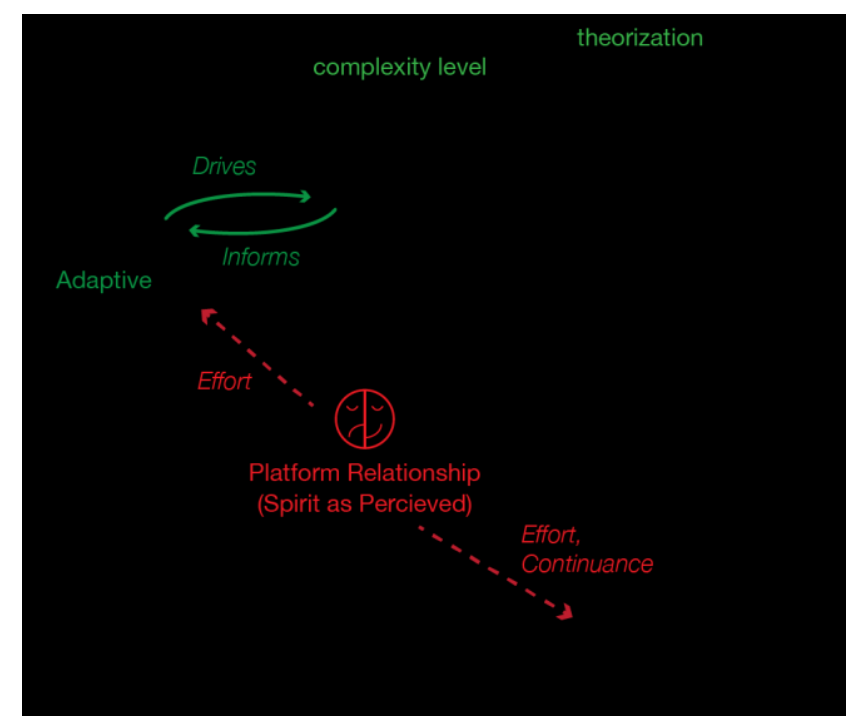

Figure 5: Updated folk theory formation process, highlighting the adaptation process itself in green and the impact of perceived platform spirit and one's relationship with the platform in red.

First, this study has updated the model of folk theorization in this context (Figure 1) to account for the change over time which is endemic to platforms [38] (the revised Figure 5). While I do not account for users who have no algorithmic awareness (potentially a sizeable group $[18,88])$, I do find that those who are aware of algorithmic influence appear to notice a 
Table 1: Folk Theorization Complexity Levels and Related Adaptation Pathways in the Context of SelfPresentation on Algorithmically-Driven Social Platforms

\begin{tabular}{|c|c|c|c|c|c|c|}
\hline \multirow{2}{*}{\multicolumn{2}{|c|}{$\begin{array}{l}\text { Theorization } \\
\text { Complexity } \\
\text { Level (TCL) }\end{array}$}} & \multirow{3}{*}{$\begin{array}{l}\begin{array}{c}\text { Minimum } \\
\text { Demonstrated } \\
\text { Theory } \\
\text { Complexity } \\
\text { (see section 4.1) }\end{array} \\
\text { Indicates the } \\
\text { theorizer is aware } \\
\text { of the algorithmic } \\
\text { process and } \\
\text { believes they have } \\
\text { identified multiple } \\
\text { criteria by which it } \\
\text { makes decisions as } \\
\text { well as causal } \\
\text { ordering within } \\
\text { this criteria (e.g., a } \\
\text { specified decision- } \\
\text { making pathway } \\
\text { or complex } \\
\text { rankings of } \\
\text { criteria). }\end{array}$} & \multirow{2}{*}{$\begin{array}{l}\text { Simplified } \\
\text { Example }\end{array}$} & \multirow{2}{*}{$\begin{array}{c}\text { Common } \\
\text { Info } \\
\text { Strategy } \\
\text { (see section } \\
4.4)\end{array}$} & \multicolumn{2}{|c|}{$\begin{array}{c}\text { Common Adaptation } \\
\text { Behavior } \\
\text { (see sections } 4.3 \text { and } 4.4 \text { ) }\end{array}$} \\
\hline & & & & & Spirit & Spirit \\
\hline \multirow[t]{2}{*}{$\underset{D}{\overparen{E}}$} & 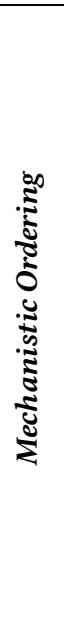 & & $\begin{array}{l}\text { Platform decides } \\
\text { what content to } \\
\text { show me by } \\
\text { filtering to only } \\
\text { posts from close } \\
\text { friends, and then } \\
\text { from that pool } \\
\text { chooses based on } \\
\text { how often I've } \\
\text { commented on } \\
\text { this person's } \\
\text { posts (important) } \\
\text { and how many } \\
\text { likes the new post } \\
\text { has (less } \\
\text { important). }\end{array}$ & \multirow{2}{*}{$\begin{array}{l}\text { Broad } \\
\text { expansion \& } \\
\text { addition of } \\
\text { exogenous } \\
\text { sources, } \\
\text { significant, } \\
\text { often creative } \\
\text { experimentati } \\
\text { on }\end{array}$} & \multirow{2}{*}{$\begin{array}{l}\text { Continuance, } \\
\text { faithful } \\
\text { adaptation } \\
\text { directed at } \\
\text { algorithmic } \\
\text { components } \\
\text { specifically } \\
\text { via } \\
\text { significant, } \\
\text { often } \\
\text { experimental, } \\
\text { modification } \\
\text { of prior self- } \\
\text { presentation } \\
\text { tactics }\end{array}$} & \multirow{2}{*}{$\begin{array}{l}\text { If platform } \\
\text { unessential, } \\
\text { leave. } \\
\text { If platform } \\
\text { essential, } \\
\text { severely limit } \\
\text { participation } \\
\text { If heavily } \\
\text { attached to } \\
\text { platform, } \\
\text { unfaithfully } \\
\text { adapt }\end{array}$} \\
\hline & 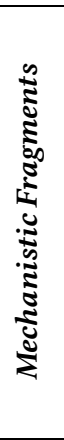 & $\begin{array}{l}\text { Indicates the } \\
\text { theorizer is aware } \\
\text { of the algorithmic } \\
\text { process and } \\
\text { believes they have } \\
\text { identified multiple } \\
\text { criteria by which it } \\
\text { makes decisions, } \\
\text { which may vary in } \\
\text { weight. }\end{array}$ & $\begin{array}{l}\text { Platform } \\
\text { considers the } \\
\text { amount of likes } \\
\text { on a post, what } \\
\text { time it was } \\
\text { posted, and how } \\
\text { close I am to the } \\
\text { person that } \\
\text { posted it when } \\
\text { deciding what to } \\
\text { show me. }\end{array}$ & & & \\
\hline \multirow{2}{*}{ : } & 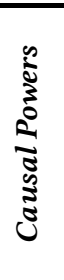 & $\begin{array}{l}\text { Indicates the } \\
\text { theorizer is aware } \\
\text { that the } \\
\text { algorithmic } \\
\text { process has some } \\
\text { specific causal } \\
\text { effect. }\end{array}$ & $\begin{array}{l}\text { Platform picks } \\
\text { what kind of } \\
\text { content to show } \\
\text { me on my feed. }\end{array}$ & \multirow{2}{*}{$\begin{array}{l}\text { Limited } \\
\text { expansion } \\
\text { and addition } \\
\text { of exogenous } \\
\text { info \& limited } \\
\text { testing } \\
\text { behavior }\end{array}$} & \multirow{2}{*}{$\begin{array}{l}\text { Continuance, } \\
\text { non- } \\
\text { algorithm- } \\
\text { specific } \\
\text { adjustment of } \\
\text { existing self- } \\
\text { presentation } \\
\text { tactics }\end{array}$} & \multirow{2}{*}{$\begin{array}{l}\text { If platform } \\
\text { unessential, } \\
\text { leave. } \\
\text { If essential, } \\
\text { limiting of } \\
\text { participation }\end{array}$} \\
\hline & 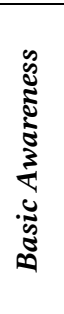 & $\begin{array}{l}\text { Indicates that the } \\
\text { theorizer is aware } \\
\text { of the presence of } \\
\text { an on-platform } \\
\text { algorithmic / } \\
\text { computational } \\
\text { mechanisms with } \\
\text { nonspecific effect. }\end{array}$ & $\begin{array}{l}\text { Platform has } \\
\text { some systems that } \\
\text { make decisions } \\
\text { for you. }\end{array}$ & & & \\
\hline
\end{tabular}


great deal of relevant algorithmic changes, but have difficulty bracketing them as algorithmic change in particular. From there the process plays out largely as described by DeVito et al. [25] with the addition of a continuous interplay between adaptive sensemaking and information foraging (Figure 5, relevant updates in green) to account for the kind of cross-checking and comparative verification that seen throughout our data, but especially among structural theorizers. Now, foraging and sensemaking are a crucial mini-loop in the process, with foraging informing adaptive sensemaking, and the midlevel products of sensemaking (preliminary folk theories) driving further sensemaking during the verification process. Notably, this cognitive loop is likely a good thing that could be encouraged via design, as repeated discussion or consideration of folk information helps expand our related theorization ability [92, 94].

Second, as theorized by DeVito et al., the process of folk theory formation is always embedded within and responsive to a larger operating context and technical environment [25] here, the process of self-presentation. While that work accounted for the influence of classic self-presentation factors such as self-presentation goals and styles [3], as well as classic selfpresentation-related individual characteristics such as web skills, self-monitoring ability, and personality factors $[24,25]$, it does not account for one's capability to undertake the process under study: folk theorization itself. My results suggest that folk theorization capacity has impacts throughout the process of folk theorization in service of self-presentation, from the types of information one is likely to base their theorization on, to the breadth of options one has in determining how to respond to platform change. Structural theorizers appear to have the advantage in this process, making this an important factor to account for in future selfpresentation and folk theories work.

Third, by centering my analysis on user perception, I have identified an important difference in adaptation processes between the organizational and personal, social platform contexts: the conflation of organizational notions of spirit and what is often referred to as task-technology fit. As Lapointe and Rivard note, decisions around whether to adapt to or resist implementation of technology are often based on the user's assessment of the match between what's new and the technology's place in the current organizational or personal setting [65]. In an organizational context, tasks are business-related and have limited personal valence; by and large, failing to complete a spreadsheet for one's employer does not represent a direct threat to one's identity and personal concerns. However, in a personal context, this does not appear to hold, as tasks can be quite personal, e.g. maintaining lines of communication with distant relatives. There are some hints of this in past folk theories work, specifically DeVito et al.'s work on \#RIPTwitter, where users frequently "articulated an already-formed expectation of how Twitter 'should' perform relative to their use case," essentially an "ad-hoc assessment of task/technology fit" which was tied directly into a sense of what a platform is for and what it had promised [26 $\mathrm{p}$. 3168]. In other words, users seemed to see disruptions to task-technology fit for personal tasks as an explicit violation of what might fairly be called the spirit of a platform.

In the present study, this linkage of task-technology fit and spirit was universal and explicit, motivating the merged concept of platform spirit. This expanded concept let me look at the impacts of how much a platform is perceived as honoring or violating this user-perceived spirit on the folk theorization and self-presentation processes, revealing the importance of accounting for perceived platform spirit when studying these topics, as it can potentially impact how much effort a user is willing to put into adaptation and sensemaking, and when violated can negatively impact basic decisions about continuance (Figure 5, relevant updates in red). 
Importantly, these results suggest that, during the adaptation process, users are not simply thinking about mechanism, nor are they simply evaluating whether a discrete change honors or violates platform spirit. Rather, these participants often took both the immediate change's impact on spirit and the platform's recent history regarding honoring or violating spirit into account. This suggests that there is a longer-term issue at play here, which may potentially constitute trust (or distrust) in a platform to honor spirit. This can, in turn, color how users interpret individual changes. Past work establishes that user perceptions of past system performance impact user trust in an algorithmically-driven system more broadly [28, 115], as does the user's sense of if a system intends to help them accomplish their goals $[67,115]$, and a version of this appears to be playing out while evaluating spirit.

While I have only used it as an example sparingly in order to illustrate the diversity of user concerns, many in my pool of queer participants brought up the Tumblr adult content ban as an example of spirit violation at some point, in conjunction with changes that were not always directly related to this change. For many of these participants, Tumblr was a crucially important platform for identity reasons - unsurprising for a platform that has been written about in academic terms as a specifically queer platform which provided a unique and crucial space for exploring the importance of the erotic in the queer community [47]. Removing adult content via algorithmic content moderation was a clear violation of platform spirit, both in the traditional platform values sense as well as in the now-emotionally-valanced use case sense, and this went on to color later evaluations of the platform in many cases. What impacts folk theorization is not just if a change honors or violates platform spirit, but rather this judgement as heavily informed by the context of a larger history of honor or violation. In fact, there are confirmatory echoes of this phenomena in related work on professional and influencer populations. Both Cotter [17] and $\mathrm{Wu}$ et al. [114] discuss situations where the systems in question (Instagram and YouTube, respectively) create a sense that they are not on the user in question's side, resulting in the kind of gaming behavior seen in unfaithful adaptation. Considering the impact of spirit violations seen in this study, perceived platform spirit represents another crucial area of future inquiry, as it is very possible I have not yet uncovered the extent of the impacts of these violations on folk theorization or user self-presentation behavior.

\subsection{Implications for Algorithmic Literacy}

Using folk theorization as a lens, this project was designed to define and provide points of intervention for algorithmic literacy. However, it is important to recognize that algorithmic literacy alone is not enough to ensure this in a broader sense. What is needed here is a New Literacy, or a literacy that concerns "the skills, strategies, and dispositions necessary to successfully use and adapt to the rapidly changing information and communication technologies and contexts that continuously emerge in our world and influence all areas of our personal and professional lives" [71 p. 1572]. New Literacies, even more so than traditional literacies, are about extensibility, as they are built to recognize that technology will change rapidly and shift in context between cultures, and aim to train people to critically evaluate and respond to situations in the field, key concerns I have laid out as motivation for this work. Ultimately, all users will still need to be able to critically evaluate content on the platform [54], and media literacy could even help one evaluate the veracity of information during exogenous information foraging. Algorithmic literacy should not stand alone, and in fact is best seen as a component of a larger platform literacy that encompasses all of the aforementioned literacies, 
with algorithmic literacy taking its place as a component literacy focused on the specific platform content and its related pace of change.

As to algorithmic literacy itself, recall my definition from earlier:

the capacity and opportunity to be aware of both the presence and impact of algorithmically-driven systems on self- or collaboratively-identified goals, and the capacity and opportunity to crystalize this understanding into a strategic use of these systems to accomplish said goals

To truly act strategically in a constantly changing environment, users must strategically adapt. To do this, users must be responsive to the causes of the effects of algorithmic platform mechanisms, not simply the effects themselves. In terms of folk theorization, these results suggest they need to be structural theorizers, working at a mechanistic, or structural, level, adapting their behavior directly to the algorithmic system itself. Looking at the structural theorizer adaptation pathway for self-presentation, it seems that the ability to work at this level enables this kind of strategic adaptation to pursue one's goals, while the functional theorists are left to simply react to effects. Moreover, the ability to theorize at a structural level appears to give the user far more freedom to experiment and expand their own knowledge, and even resist through unfaithful adaptation in some cases. Structural theorization ability appears to give the user agency. In turn, a sense of agency prompts more useful learning and creative adaptation as opposed to the stagnation those with less agency may find [6].

From a folk theorization perspective, the goal in terms of reaching algorithmic literacy is to boost users to structural theorization. There is significant reason to think this is possible, as functional and structural theorization respectively deal with functional and structural knowledge, and the former can help build the latter. While functional knowledge, e.g. knowledge that reaches the level of causal powers, can't tell us how a system works, it does serve to inform us where complexity likely exists and merits further explanation [60]. Humans regularly infer the degree of internal complexity of a system by examining the external functionality [2]. Functional knowledge, especially causal powers-level theorizing, can be turned to structural knowledge/mechanistic theorizing, which in turn enables the strategic, effective use I would call algorithmic literacy.

To be clear, by "understanding structure" in this context, I do not mean full mechanistic knowledge, or even the highest-level mechanistic ordering-style theories. This study revealed few significant differences within structural theorization, and none that would indicate mechanistic ordering is necessary for algorithmic literacy. Rather, the threshold appears to be knowledge that multiple mechanistic fragments are part of the algorithmic decision-making process in question. In fact, it may not matter if knowledge of specific fragments is retained for long. Having mechanistic knowledge, even temporarily, creates memory and structure of complexity [2, 61]. In terms of practical on-platform adaptation, this suggests that even if specific knowledge of specific fragments is not retained, the knowledge that there are many fragments is enough to tell the user how to adapt: ramp up information gathering to find out what has changed in terms of the influence of various mechanistic fragments.

\subsubsection{Future Work: How to Boost Theorization}

Future work in this area should explore possible mechanisms for introducing this more structural information to functional theorizers. To begin with, the fact that there is no need for retention of specific structural information suggests that informal approaches in line with the 
larger multiliteracies approach would be appropriate and effective here [64, 71]. In terms of more formal approaches, I propose two avenues of future exploration.

One approach, largely borrowed from the cognitive development literature, is to carefully scaffold causal understanding via interrogating mechanistic detail. For true conceptual change to occur, a discrete process of what is essentially knowledge-based infrastructure building is necessary in order to create the spaces which will be filled in by new knowledge [113]. In order to build this mechanistic scaffold, one must open the individual up to questioning their current theory.

Another potential approach is to provide useful experts. Humans are largely wired to defer to relevant experts when needed [59]. Especially if we can impress causal complexity onto users as discussed above, allowing them to better know when to turn to an expert, the recruitment or creation of an expert source could be valuable.

\subsection{Challenges for Platforms}

This study suggests four direct challenges to social platforms themselves, which I hope platforms will be mindful of and attempt to directly address in future design work. First, as noted in the results, the crucial noticing step that kicks off adaptation seems to be hampered by repeated conflation of change type, such that feature changes appear to crowd out opportunities to notice algorithmic change. This is essentially a problem with bracketing, the step in sensemaking which involves bounding the location and extent of the change phenomena in order to begin assessing and adapting to the correct change in the correct manner [110]. To address this noticing challenge, one approach is to use the problem to the platform's advantage, pairing more subtle algorithmic changes with very visual interface or aesthetic changes as a signaling mechanism. On a larger scale, approaches such as seamful design could be considered $[31,89]$.

Second, this study highlights the importance of platforms understanding not just simple user satisfaction, but the full relationship and related expectations encapsulated in platform spirit and the platform's history of honoring or violating that spirit. To borrow terms from Gillespie's definitions of "algorithm," platforms must account for the user's conception of them as both synecdoche (the entire technical system and related inputs, outputs, and value system) and talisman (an agential actor representing the platform and its ownership) [39]. Based on these findings, prior work in this context (e.g., [26]), and work on user understanding more generally (e.g., [37, 53]), it seems clear that users are holding the platform accountable to their own perceptions of spirit as they would hold any agential actor accountable. As such, they want to understand not just the "what" technically, but the "why" behind platform actions, likely viewing (and judging) the platform as a fellow social actor [68, 98]. Platforms that ignore spirit and especially those that repeatedly violate what their users perceive as their spirit risk the detrimental trust- and sensemaking-related problems discussed at the top of section 5 , as well as use-related consequences. These results suggest that spirit-based judgments of platforms are a major basis upon which people decide if they will adapt. They also suggest that limiting one's participation to the point of being a user who is far less useful to the platform or simply leaving are definitely alternatives on the table.

The third challenge is also a charge: never forget that adaptation to change on social platforms is labor, and act accordingly when designing, deploying, and explaining new versions of systems. Platforms would be well advised to not take adaptation as a given. Rather, platforms are advised to treat ability (or willingness) to adapt as a finite resource. At a base level, 
considering that nearly all participants repeatedly took issue with the pace of change, this includes being very attentive to and internally critical about what truly needs to change about a platform. To go further, platforms can consider ways to address the barriers to getting the information needed to make adaptation decisions discussed in section 4.4.4, which broadly concern a lack of specificity for all, a lack of simplicity for functional theorizers, and an organizational challenge for structural theorizers. To tackle specificity while being mindful that they are asking for labor, platforms are advised to make the value proposition of adapting very clear to the user, as adaptation to technology more generally has been shown to be positively affected by making the personal relevance and specific utility of a change clear $[22,29,36,81$, $106,107]$. To tackle the clarity problems faced by functional theorizers, platforms can attend to how they explain, with repetition and elaboration [93] and the use of storytelling techniques and user-congruent framing $[21,53,90]$ as possible mechanisms and the emerging body of work on algorithmic explanation as a guide (for a full treatment, see [87]). To tackle the organizational problems faced by structural theorizers, I forward the concept of a change clearinghouse from several of the participants, a central location for notification and librarystyle reference on social platform change - essentially, a platform-built exogenous foraging aid.

Of course, improved knowledge of the platform in the first place would aid adaptation overall, from initial noticing to adaptive sensemaking [59, 99, 100, 109], which is why the final challenge is to find ways to support algorithmic literacy both on and off platform. To be clear, I put this responsibility squarely on platforms, not individual users or even educators. It is instructive here to look to the case of media literacy, where a focus on personal responsibility left the task of assessing and boosting literacy largely with individuals, parents, and schools, resulting in overestimation of skills and limited progress in the overall effort to build media literacy [12]. Simply put, this cannot be left to the individual, or done on a casual catch-ascatch-can basis. Both approaches to past literacies have resulted in a lack of progress as well as specific deficits and growing inequality for low-resourced individuals [8, 12, 54, 56, 109], the very same groups that are now showing an emerging gap around algorithmic knowledge [18]. Consider the outsized influence platforms have over work, information flow, politics, and sociality [38, 40, 69, 82, 83], juxtaposed against the apparent importance of endogenous information as a folk theorization source for functional theorizers especially, the need for nonclassroom, informal intervention with any New Literacy [71], and the sheer amount of resources most platform companies currently hold. Clearly, platforms are in an ideal place structurally and financially to take the lead on algorithmic literacy.

\subsection{Limitations, Transferability, and Future Work}

This study must be read within the context of the project's limitations. Additionally, as an exploratory study, it only partially answers some important, emerging questions. In addition to the limitations regarding transferability discussed in section 5.3.1, there are three notable areas for caution in interpretation and future work.

First, though section 3.2.1 spells out my rationale for using Facebook as an organizing platform, and my findings provide an important picture of how theorization and literacy appear to work in the current platform environment, they are still ultimately limited by the bounds of that environment. Future work should explore how theorization does and can happen in algorithmic spaces beyond dominant platforms. Considering the continued and sometimesheightened importance of exogenous information to the theorization process, and the impact of perceived platform spirit on adaptation, it would be particularly interesting to explore how 
these processes work in spaces where the community itself helps set algorithmic standards, e.g., peer production and open source environments.

Second, while my findings around the impact of perceived platform spirit on adaptation provide a way to begin accounting for affective and trust-based aspects of the human/platform relationship, future work is needed to better understand what informs perceived spirit. Considering how often participants discussed not being able to leave platforms, and how important individual use case appears to be to perceived platform spirit, future work is needed which more thoroughly explores theorization, adaptation, and literacy in the context of one's overall personal social media ecosystem and use history.

Third, while this study engaged participants deeply on one area of their technology use, it did not fully account for the individual's overall baseline relationship to technology. Considering the enjoyment expressed by some participants over their adaptations, juxtaposed against the sheer annoyance felt by others at the thought of even having to adapt, it is possible that high-level relationship to technology plays a yet-unspecified part in adaptation. Future work which more thoroughly explores use history beyond social platforms, voluntary exploratory behavior around technology, and propensity to test or tinker with systems could help further refine our understanding.

\subsubsection{Transferability Beyond Queer Populations}

It is important to examine one's qualitative findings in the context of the situation and population they stem from in order to assess transferability [45]. Upon examination, I remain confident that these results are broadly transferrable, with a few important caveats. First, I inadvertently failed to sample those who are extremely closeted - as such, it is possible that I have not adequately explored those who are not open about their identities in general or who have extremely salient reasons not to disclose their identities. This is an important area for future work.

Second, it is important to note that the majority of participants used the Tumblr adult content ban as an example at some point. While this is unsurprising due to the sheer importance of Tumblr to queer populations [47] and the outsized negative impact of this particular change on queer identity development, it does suggest that the queer population as a whole may have more reason to be aware of algorithmic influences on platforms. However, precipitating events are not unique to the queer community, as seen in \#RIPTwitter [26]. While this does not directly threaten the transferability of our results, it likely resulted in a sample that is overall more aware of algorithmic actors than a general population sample. It is likely that more users than indicated here are either at pre-awareness or a functional TCL, highlighting both the importance of future work on interventions to boost theorization capacity and the need for continued work on fostering initial algorithmic awareness.

\subsubsection{Future Work: Single Mechanistic Fragment Theorists}

In addition to these limitations, I also chose not to report out on a small group of participants who demonstrate mechanistic understandings of platforms, but insist that only one factor or mechanistic fragment drives algorithmic decision making. I did not have enough of these participants to adequately examine and thoroughly report on this type of understanding, but preliminarily this group seems to have trouble detecting algorithmic change, an overreliance on endogenous information, difficulty taking action to adapt, and an overall feeling of inefficacy 
and helplessness. Further work directly with this group is needed to better understand them while also identifying ways to help move past any potentially harmful dig-in effect.

\section{CONCLUSION}

This study has expanded our knowledge of the folk theorization and adaptation process in the context of self-presentation on social platforms. In doing so, it has provided guidance for an algorithmic literacy that accounts for the constantly-changing platform landscape. By studying not just user folk theories, but rather their entire process of folk theorization, I was able to identify future points of intervention for this literacy, as well as potential paths forward. My hope is that by starting in the user's own perceptions and lived experiences, at the level of the user's own folk theorization, we can promote the virtuous relationship between functional and structural knowledge which can build literacy, helping users to theorize, to "work with" the structure of platforms to pursue their goals, even in the face of the unexpected.

\section{ACKNOWLEDGMENTS}

This work was supported by a Graduate Research Grant from The Graduate School at Northwestern University, and was originally part of my dissertation. I thank my dissertation committee - Jeremy Birnholtz, Jeff Hancock, Darren Gergle, and Karrie Karahalios - for their commentary on earlier versions of this work. I also thank Stevie Chancellor, Sarah Gilbert, and Ashley Marie Walker for their commentary on earlier versions, as well as their support during the entire research process. Additionally, I thank the reviewers and associate chairs for their commentary on the work. I acknowledge the invaluable research assistance of Amanda Davis, Samantha Hassett, and Samantha Zhang. Finally, I thank my participants for their time, and for sharing their experiences, without which this work would not be at all possible.

\section{REFERENCES}

[1] ACM US Public Policy Council. 2017. Statement on Algorithmic Transparency and Accountability. Retrieved from https://www.acm.org/binaries/content/assets/public-policy/2017_usacm_statement_algorithms.pdf

[2] Richard E Ahl and Frank C Keil. 2016. Diverse Effects, Complex Causes: Children Use Information About Machines' Functional Diversity to Infer Internal Complexity. Child Development, 88, 3: 828-845.

[3] Robert M Arkin. 1981. Self-Presentation Styles. In Impression Management Theory and Social Psychological Research, James T Tedeschi (ed.). Academic Press, New York, NY, 311-334.

[4] Shaowen Bardzell and Jeffrey Bardzell. 2011. Towards a feminist HCI methodology: social science, feminism, and HCI. In Proceedings of the SIGCHI Conference on Human Factors in Computing Systems, 675-684.

[5] Eric PS Baumer and Jed R Brubaker. 2017. Post-userism. In Proceedings of the 2017 CHI Conference on Human Factors in Computing Systems, 6291-6303.

[6] Anne Beaudry and Alain Pinsonneault. 2005. Understanding user responses to information technology: A coping model of user adaptation. MIS Quarterly, 29, 3: 493-524.

[7] David Beer. 2009. Power through the algorithm? Participatory web cultures and the technological unconscious. New Media \& Society, 11, 6: 985-1002.

[8] Shirley J. Behrens. 1994. A conceptual analysis and historical overview of information literacy. College \& Research Libraries, 55, 4: 309-322.

[9] Michael S. Bernstein, Eytan Bakshy, Moira Burke, and Brian Karrer. 2013. Quantifying the invisible audience in social networks. In Proceedings of the SIGCHI Conference on Human Factors in Computing Systems, 21-30.

[10] Courtney Blackwell, Jeremy Birnholtz, and Charles Abbott. 2015. Seeing and being seen: Co-situation and impression formation using Grindr, a location-aware gay dating app. New media \& society, 17, 7: 1117-1136.

[11] Lisa Bowleg. 2013. "Once you've blended the cake, you can't take the parts back to the main ingredients": Black gay and bisexual men's descriptions and experiences of intersectionality. Sex Roles, 68, 11-12: 754-767.

[12] Monica Bulger and Patrick Davison. 2018. The Promises, Challenges, and Futures of Media Literacy. Data \& Society.

[13] John T Cacioppo and Richard E Petty. 1982. The need for cognition. Journal of personality and social psychology, 42, 1: 116. 
[14] John M Carrol. 1999. Five Reasons for Scenario-Based Design. In Proceedings of the 32nd Annual Hawaii International Conference on Systems Sciences, 1-11.

[15] Kathy Charmaz. 2006. Constructing Grounded Theory: A Practical Guide Through Qualitative Analysis. Sage, Thousand Oaks, CA.

[16] Mustapha Cheikh-Ammar. 2018. The IT artifact and its spirit: a nexus of human values, affordances, symbolic expressions, and IT features. European fournal of Information Systems, 27, 3: 278-294.

[17] Kelley Cotter. 2018. Playing the visibility game: How digital influencers and algorithms negotiate influence on Instagram. New Media \& Society, 21, 4: 895-913.

[18] Kelley Cotter and Bianca C. Reisdorf. 2020. Algorithmic Knowledge Gaps: A New Dimension of (Digital) Inequality. International fournal of Communication, 14, 2020: 745-765.

[19] Andrea Daley, Judith A. MacDonnell, Shari Brotman, Melissa St Pierre, Jane Aronson, and Loralee Gillis. 2017. Providing health and social services to older LGBT adults. Annual Review of Gerontology \& Geriatrics, 37, 143.

[20] Cathy N. Davidson. 2012. A fourth " $r$ " for 21st century literacy. The Washington Post, Washington, DC.

[21] Elizabeth Davidson. 2006. A technological frames perspective on information technology and organizational change. The fournal of Applied Behavioral Science, 42, 1: 23-39.

[22] Fred D. Davis. 1989. Perceived usefulness, perceived ease of use, and user acceptance of information technology. MIS Quarterly, 13, 3: 319-340.

[23] Gerardine DeSanctis and Marshall Scott Poole. 1994. Capturing the complexity in advanced technology use: Adaptive structuration theory. Organization Science, 5, 2: 121-147.

[24] Michael Ann DeVito, Jeremy Birnholtz, and Jeffrey T. Hancock. 2017. Platforms, People, and Perception: Using Affordances to Understand Self-Presentation on Social Media. In Proceedings of the 2017 ACM Conference on Computer Supported Cooperative Work and Social Computing, 740-754.

[25] Michael Ann DeVito, Jeremy Birnholtz, Jeffrey T. Hancock, Megan French, and Sunny Liu. 2018. How People Form Folk Theories of Social Media Feeds and What It Means for How We Study Self-Presentation. In Proceedings of the 2018 CHI Conference on Human Factors in Computing Systems, Paper 120.

[26] Michael Ann DeVito, Darren Gergle, and Jeremy Birnholtz. 2017. “Algorithms ruin everything”: \#RIPTwitter, Folk Theories, and Resistance to Algorithmic Change in Social Media. In Proceedings of the 2017 CHI Conference on Human Factors in Computing Systems, 3163-3174.

[27] Michael Ann DeVito, Ashley M. Walker, and Jeremy Birnholtz. 2018. "Too Gay for Facebook:" Presenting LGBTQ+ Identity Throughout the Personal Social Media Ecosystem. Proceedings of the ACM on Human-Computer Interaction, 2, CSCW: 44.

[28] Berkeley J. Dietvorst, Joseph P. Simmons, and Cade Massey. 2015. Algorithm aversion: People erroneously avoid algorithms after seeing them err. Journal of Experimental Psychology: General, 144, 1: 114.

[29] Andrea A. DiSessa. 2001. Changing minds: Computers, learning, and literacy. MIT Press, Cambridge, MA.

[30] Nicole B Ellison, Charles Steinfield, and Cliff Lampe. 2007. The benefits of Facebook "friends:" Social capital and college students' use of online social network sites. Journal of Computer-Mediated Communication, 12, 4: 11431168 .

[31] Motahhare Eslami, Karrie Karahalios, Christian Sandvig, Kristen Vaccaro, Aimee Rickman, Kevin Hamilton, and Alex Kirlik. 2016. First I "like" it, then I hide it: Folk Theories of Social Feeds. In Proceedings of the 34rd Annual SIGCHI Conference on Human Factors in Computing Systems, 2371-2382.

[32] Motahhare Eslami, Aimee Rickman, Kristen Vaccaro, Amirhossein Aleyasen, Andy Vuong, Karrie Karahalios, Kevin Hamilton, and Christian Sandvig. 2015. "I always assumed that I wasn't really that close to [her]": Reasoning about invisible algorithms in the news feed. In Proceedings of the 33rd Annual SIGCHI Conference on Human Factors in Computing Systems, 153-162.

[33] Laura Boyd Farmer and Rebekah Byrd. 2015. Genderism in the LGBTQQIA community: An interpretative phenomenological analysis. fournal of LGBT Issues in Counseling, 9, 4: 288-310.

[34] Megan French and Jeff T. Hancock. 2017. What's the Folk Theory? Reasoning About Cyber-Social Systems. Paper presented at the 67th Annual Confrence of the International Communication Association (San Diego, CA).

[35] Megan French, Jeff T. Hancock, Sunny Liu, Michael Ann DeVito, and Jeremy Birnholtz. 2018. Confronting the unexpected: The nature and effects of attributing responsibility to social media platforms for expectancy violations. Paper presented at the 68th Annual Conference of the International Communication Association (Prague, Czech Republic).

[36] Helene Gelderblom and Leanne Menge. 2018. The invisible gorilla revisited: using eye tracking to investigate inattentional blindness in interface design. In Proceedings of the 2018 International Conference on Advanced Visual Interfaces, 39.

[37] Susan A. Gelman and Cristine H. Legare. 2011. Concepts and folk theories. Annual Review of Anthropology, 40379398. 
[38] Tarleton Gillespie. 2014. The Relevance of Algorithms. In Media Technologies: Essays on Communication, Materiality, and Society, Tarleton Gillespie, Pablo Boczkowski and Kirsten Foot (ed.). MIT Press, Cambridge, MA, 167-193.

[39] Tarleton Gillespie. 2016. Algorithm. In Digital Keywords: A Vocabulary of Information Society and Culture, Benjamin Peters (ed.). Princeton University Press, Princeton, NJ, 18-30.

[40] Tarleton Gillespie. 2018. Custodians of the Internet: Platforms, content moderation, and the hidden decisions that shape social media. Yale University Press, New Haven, CT.

[41] Tarleton Gillespie. 2018. Platforms are not intermediaries. Georgetown Law Technology Review, 2, 2: 198-216.

[42] Erving Goffman. 1959. The presentation of self in everyday life. Anchor, New York, NY.

[43] Erving Goffman. 1963. Stigma: Notes on the management of spoiled identity. Simon and Schuster, New York, NY.

[44] Mary L Gray. 2009. Out in the country: Youth, media, and queer visibility in rural America. NYU Press, New York, NY.

[45] Egon G Guba and Yvonna S Lincoln. 1982. Epistemological and methodological bases of naturalistic inquiry. ECTf, 30, 4: 233-252.

[46] Michael Gurstein. 2003. Effective use: A community informatics strategy beyond the digital divide. First Monday, 8, 12 .

[47] Oliver L Haimson, Avery Dame-Griff, Elias Capello, and Zahari Richter. 2021. Tumblr was a trans technology: the meaning, importance, history, and future of trans technologies. Feminist Media Studies, 21, 3: 345-361.

[48] Sandra G Harding. 2004. Introduction: Standpoint theory as a site of political, philosophic, and scientific debate. In The Feminist Standpoint Theory Reader: Intellectual and Political Controversies, Sandra G Harding (ed.). Routledge, 1-15.

[49] Jean Hardy and Silvia Lindtner. 2017. Constructing a desiring user: Discourse, rurality, and design in locationbased social networks. In Proceedings of the 2017 ACM Conference on Computer Supported Cooperative Work and Social Computing, 13-25.

[50] Eszter Hargittai. 2002. Second-Level Digital Divide: Differences in People's Online Skills. First Monday, 7, 4.

[51] Eszter Hargittai, Jonathan Gruber, Teodora Djukaric, Jaelle Fuchs, and Lisa Brombach. 2020. Black box measures? How to study people's algorithm skills. Information, Communication \& Society, 23, 5: 764-755.

[52] Eszter Hargittai and Yuli Patrick Hsieh. 2012. Succinct survey measures of web-use skills. Social Science Computer Review, 30, 1: 95-107.

[53] David Herman. 2013. Storytelling and the Sciences of Mind. MIT Press, Cambridge, MA.

[54] Henry Jenkins, Ravi Purushotma, Margaret Weigel, Katie Clinton, and Alice J Robison. 2009. Confronting the challenges of participatory culture: Media education for the 21st century. MIT Press, Cambridge, MA.

[55] Shagun Jhaver, Yoni Karpfen, and Judd Antin. 2018. Algorithmic Anxiety and Coping Strategies of Airbnb Hosts. In Proceedings of the 2018 CHI Conference on Human Factors in Computing Systems, 421.

[56] Genevieve Marie Johnson. 2007. Functional Internet literacy: Required cognitive skills with implications for instruction. E-Learning and Digital Media, 4, 4: 433-441.

[57] Jeffrey C Johnson and Susan C Weller. 2002. Elicitation techniques for interviewing. In Handbook of interview research: Context and method, Jaber F Gubrim and James A Holstein (ed.). Sage, Thousand Oaks, CA, 491-514.

[58] Frank C. Keil. 2003. Folkscience: Coarse interpretations of a complex reality. Trends in Cognitive Sciences, 7, 8: 368-373.

[59] Frank C. Keil. 2010. The feasibility of folk science. Cognitive Science, 34, 5: 826-862.

[60] Frank C. Keil. 2012. Does folk science develop? In The journey from child to scientist: Integrating cognitive development and the education sciences, Sharon M. Carver and Jeff Shrager (ed.). American Psychological Association, Washington, DC, 67-86.

[61] Frank C. Keil. 2012. Running on empty? How folk science gets by with less. Current Directions in Psychological Science, 21, 5: 329-334.

[62] Douglas Kellner and Jeff Share. 2005. Toward critical media literacy: Core concepts, debates, organizations, and policy. Discourse: studies in the cultural politics of education, 26, 3: 369-386.

[63] Erin Klawitter and Eszter Hargittai. 2018. "It's Like Learning a Whole Other Language:" The Role of Algorithmic Skills in the Curation of Creative Goods. International fournal of Communication, 12, 2018: 3490-3510.

[64] Michele Knobel and Colin Lankshear. 2014. Studying new literacies. Journal of adolescent \& adult literacy, 58, 2 97-101.

[65] Liette Lapointe and Suzanne Rivard. 2005. A multilevel model of resistance to information technology implementation. MIS Quarterly, 29, 3: 461-491.

[66] Mark R. Leary and Robin M. Kowalski. 1990. Impression management: A literature review and two-component model. Psychological bulletin, 107, 1: 34.

[67] John D. Lee and Katrina A. See. 2004. Trust in automation: Designing for appropriate reliance. Human Factors, 46, 1: 50-80. 
[68] Min Kyung Lee. 2018. Understanding perception of algorithmic decisions: Fairness, trust, and emotion in response to algorithmic management. Big Data \& Society, 5, 1.

[69] Min Kyung Lee, Daniel Kusbit, Evan Metsky, and Laura Dabbish. 2015. Working with machines: The impact of algorithmic and data-driven management on human workers. In Proceedings of the 33rd Annual ACM Conference on Human Factors in Computing Systems, 1603-1612.

[70] Richard D. Lennox and Raymond N. Wolfe. 1984. Revision of the self-monitoring scale. fournal of Personality and Social Psychology, 46, 6: 1349-1364.

[71] Donald J. Leu, Charles K. Kinzer, Julie L. Coiro, and Dana W. Cammack. 2004. Toward a theory of new literacies emerging from the Internet and other information and communication technologies. Theoretical Models and Processes of Reading, 5, 1: 1570-1613.

[72] Eden Litt, Erin Spottswood, Jeremy Birnholtz, Jeff T Hancock, Madeline E Smith, and Lindsay Reynolds. 2014. Awkward encounters of an other kind: collective self-presentation and face threat on facebook. In Proceedings of the 17th ACM Conference on Computer Supported Cooperative Work \& Social Computing, 449-460.

[73] Haley MacLeod, Ben Jelen, Annu Prabhakar, Lora Oehlberg, Katie Siek, and Kay Connelly. 2017. A Guide to Using Asynchronous Remote Communities (ARC) for Researching Distributed Populations. EAI Endorsed Transactions on Pervasive Health and Technology, 17, 11: 7.

[74] Juan F Maestre, Elizabeth V Eikey, Mark Warner, Svetlana Yarosh, Jessica Pater, Maia Jacobs, Gabriela Marcu, and Patrick C Shih. 2018. Conducting Research with Stigmatized Populations: Practices, Challenges, and Lessons Learned. In Companion of the 2018 ACM Conference on Computer Supported Cooperative Work and Social Computing, 385-392.

[75] Juan F Maestre, Haley MacLeod, Ciabhan L Connelly, Julia C Dunbar, Jordan Beck, Katie A Siek, and Patrick C Shih. 2018. Defining through expansion: conducting asynchronous remote communities (arc) research with stigmatized groups. In Proceedings of the 2018 CHI Conference on Human Factors in Computing Systems, 557.

[76] M Lynne Markus and Mark S Silver. 2008. A foundation for the study of IT effects: A new look at DeSanctis and Poole's concepts of structural features and spirit. Journal of the Association for Information systems, 9, 10: 5.

[77] Jonathan Mohr and Ruth Fassinger. 2000. Measuring dimensions of lesbian and gay male experience. Measurement and Evaluation in Counseling and Development, 33, 2: 66-66.

[78] Jonathan J Mohr and Matthew S Kendra. 2011. Revision and extension of a multidimensional measure of sexual minority identity: The Lesbian, Gay, and Bisexual Identity Scale. Journal of counseling psychology, 58, 2: 234.

[79] Philip M Napoli. 2014. Automated media: An institutional theory perspective on algorithmic media production and consumption. Communication Theory, 24, 3: 340-360.

[80] Wanda J Orlikowski and Debra C Gash. 1994. Technological frames: making sense of information technology in organizations. ACM Transactions on Information Systems (TOIS), 12, 2: 174-207.

[81] Wanda J Orlikowski and D. Hofman. 1997. An Improvisational Model of Change Management: The Case of Groupware Technologies. MIT Sloan Management Review, 38, 2.

[82] Frank Pasquale. 2015. The black box society: The secret algorithms that control money and information. Harvard University Press, Cambridge, MA.

[83] Frank A Pasquale. 2011. Restoring transparency to automated authority. fournal on Telecommunications and High Technology Law, 9, 2011: 235-254.

[84] Andrew Perrin and Monica Anderson. 2019. Share of U.S. adults using social media, including Facebook, is mostly unchanged since 2018. Pew Research Center, Washington, DC.

[85] Alina Pommeranz, Christian Detweiler, Pascal Wiggers, and Catholijn Jonker. 2012. Elicitation of situated values: need for tools to help stakeholders and designers to reflect and communicate. Ethics and Information Technology, 14, 4: 285-303.

[86] Annu Sible Prabhakar, Lucia Guerra-Reyes, Vanessa M Kleinschmidt, Ben Jelen, Haley MacLeod, Kay Connelly, and Katie A Siek. 2017. Investigating the suitability of the asynchronous, remote, community-based method for pregnant and new mothers. In Proceedings of the 2017 CHI Conference on Human Factors in Computing Systems, 4924-4934.

[87] Emilee Rader, Kelley Cotter, and Janghee Cho. 2018. Explanations as Mechanisms for Supporting Algorithmic Transparency. In Proceedings of the 36th Annual SIGCHI Conference on Human Factors in Computing Systems, Paper 103.

[88] Emilee Rader and Rebecca Gray. 2015. Understanding User Beliefs About Algorithmic Curation in the Facebook News Feed. In Proceedings of the 33rd Annual ACM Conference on Human Factors in Computing Systems, 173-182.

[89] Emilee Rader and Janine Slaker. 2017. The importance of visibility for folk theories of sensor data. In Proceedings of the Thirteenth Symposium on Usable Privacy and Security (SOUPS 2017), 257-270.

[90] Emilee Rader, Rick Wash, and Brandon Brooks. 2012. Stories as informal lessons about security. In Proceedings of the Eighth Symposium on Usable Privacy and Security, 1-17. 
[91] Lee Rainie and Janna Anderson. 2017. Code-Dependent: Pros and Cons of the Algorithm Age. Pew Research Center, Washington, DC.

[92] Nancy K. Ratner and Rose R. Olver. 1998. Reading a tale of deception, learning a theory of mind? Early Childhood Research Quarterly, 13, 2: 219-239.

[93] Benjamin M. Rottman and Frank C. Keil. 2011. What matters in scientific explanations: Effects of elaboration and content. Cognition, 121, 3: 324-337.

[94] Benjamin M. Rottman and Frank C. Keil. 2012. Causal structure learning over time: Observations and interventions. Cognitive Psychology, 64, 1-2: 93-125.

[95] Leonid Rozenblit and Frank Keil. 2002. The misunderstood limits of folk science: An illusion of explanatory depth. Cognitive Science, 26, 5: 521-562.

[96] Christian Sandvig, Kevin Hamilton, Karrie Karahalios, and Cedric Langbort. 2014. Auditing algorithms: Research methods for detecting discrimination on internet platforms. Paper presented at the 64th Annual Meeting of the International Communication Association (Seattle, WA).

[97] Morgan Klaus Scheuerman, Stacy M Branham, and Foad Hamidi. 2018. Safe spaces and safe places: Unpacking technology-mediated experiences of safety and harm with transgender people. Proceedings of the ACM on HumanComputer Interaction, 2, CSCW: 155.

[98] Kurt Schmitz, James T.C. Teng, and Kimberly Webb. 2016. Capturing the Complexity of Malleable IT Use: Adaptive Structuration Theory for Individuals. MIS Quarterly, 40, 3: 663-686.

[99] Daniel J Simons and Daniel T Levin. 1998. Failure to detect changes to people during a real-world interaction. Psychonomic Bulletin \& Review, 5, 4: 644-649.

[100] Daniel J Simons and Ronald A Rensink. 2005. Change blindness: Past, present, and future. Trends in cognitive sciences, 9, 1: 16-20.

[101] Martina Mahnke Skrubbeltrang, Josefine Grunnet, and Nicolai Traasdahl Tarp. 2017. \#RIPINSTAGRAM: Examining user's counter-narratives opposing the introduction of algorithmic personalization on Instagram. First Monday, 22, 4.

[102] Aaron Smith. 2018. Public Attitudes Toward Computer Algorithms. Pew Research Center, Washington, DC.

[103] Helen Spencer-Oatey. 2007. Theories of identity and the analysis of face. fournal of Pragmatics, 39, 4: 639-656.

[104] Ted Striphas. 2015. Algorithmic culture. European Journal of Cultural Studies, 18, 4-5: 395-412.

[105] Jan E Trost. 1986. Statistically nonrepresentative stratified sampling: A sampling technique for qualitative studies. Qualitative sociology, 9, 1: 54-57.

[106] Kami E. Vaniea, Emilee Rader, and Rick Wash. 2014. Betrayed by updates: how negative experiences affect future security. In Proceedings of the SIGCHI Conference on Human Factors in Computing Systems, 2671-2674.

[107] Viswanath Venkatesh, Michael G Morris, Gordon B Davis, and Fred D Davis. 2003. User acceptance of information technology: Toward a unified view. MIS Quarterly, 27, 3: 425-478.

[108] Ashley Marie Walker and Michael Ann DeVito. 2020. "'More gay' fits in better": Intracommunity Power Dynamics and Harms in Online LGBTQ+ Spaces. In Proceedings of the 2020 CHI Conference on Human Factors in Computing Systems, 1-15.

[109] Sheila Webber and Bill Johnston. 2000. Conceptions of information literacy: new perspectives and implications. fournal of Information Science, 26, 6: 381-397.

[110] Karl E Weick, Kathleen M Sutcliffe, and David Obstfeld. 2005. Organizing and the process of sensemaking. Organization Science, 16, 4: 409-421.

[111] Jillian Weiss. 2011. Reflective paper: GL versus BT: The archaeology of biphobia and transphobia within the US gay and lesbian community. Fournal of Bisexuality, 11, 4: 498-502.

[112] Michele Willson. 2017. Algorithms (and the) everyday. Information, Communication \& Society, 20, 1: 137-150.

[113] Marianne Wiser and Carol L Smith. 2016. How Is Conceptual Change Possible? Insights from Science Education. In Core Knowledge and Conceptual Change, D. Barner and A.S. Baron (ed.). Oxford University Press, New York, NY, 29-51.

[114] Eva Yiwei Wu, Emily Pedersen, and Niloufar Salehi. 2019. Agent, Gatekeeper, Drug Dealer: How Content Creators Craft Algorithmic Personas. Proceedings of the ACM on Human-Computer Interaction, 3, CSCW: 1-27.

[115] Kun Yu, Shlomo Berkovsky, Ronnie Taib, Dan Conway, Jianlong Zhou, and Fang Chen. 2017. User trust dynamics: An investigation driven by differences in system performance. In Proceedings of the 22nd International Conference on Intelligent User Interfaces, 307-317.

Received January 2021, revised April 2021; accepted May 2021. 